\title{
Synthesis, Structural Characterization, and Electrochemical Studies of New Oxovanadium(V) Complexes Derived from 2-Furanoylhydrazon Derivatives
}

\author{
Rahman Bikas, ${ }^{1}$ Hassan Hosseini-Monfared, ${ }^{1}$ Erwann Jeanneau, ${ }^{2}$ and Behrouz Shaabani ${ }^{3}$ \\ ${ }^{1}$ Department of Chemistry, Faculty of Science, University of Zanjan, Zanjan 45195-313, Iran \\ ${ }^{2}$ Université Lyon 1, Centre de Diffractométrie Henri Longchambon, Bâtiment 305, 43 boulevard du 11 Novembre 1918, \\ F-69622 Villeurbanne Cedex, France \\ ${ }^{3}$ Research Laboratory of Synthesis of Inorganic Compounds, Department of Inorganic Chemistry, \\ Faculty of Chemistry, University of Tabriz, Tabriz 51666, Iran \\ Correspondence should be addressed to Rahman Bikas; bikas_r@yahoo.com
}

Received 9 June 2012; Accepted 30 July 2012

Academic Editor: Gustavo Portalone

Copyright (c) 2013 Rahman Bikas et al. This is an open access article distributed under the Creative Commons Attribution License, which permits unrestricted use, distribution, and reproduction in any medium, provided the original work is properly cited.

Five monooxovanadium $(\mathrm{V})$ complexes $\left[\mathrm{VO}\left(\mathrm{L}^{1}\right)\left(\mathrm{OCH}_{3}\right)\left(\mathrm{OHCH}_{3}\right)\right] \quad(\mathbf{1}), \quad\left[\mathrm{VO}\left(\mathrm{L}^{2}\right)\left(\mathrm{OCH}_{3}\right)\left(\mathrm{OHCH}_{3}\right)\right] \quad(\mathbf{2}), \quad\left[\mathrm{VO}\left(\mathrm{L}^{3}\right)\left(\mathrm{OCH}_{3}\right)\right.$ $\left.\left(\mathrm{OHCH}_{3}\right)\right](3)$, $\left[\mathrm{VO}\left(\mathrm{L}^{4}\right)\left(\mathrm{OCH}_{3}\right)\left(\mathrm{OHCH}_{3}\right)\right](4)$, and $\left[\mathrm{VO}\left(\mathrm{L}^{5}\right)\left(\mathrm{OCH}_{3}\right)\left(\mathrm{OHCH}_{3}\right)\right](5)$ were synthesized and characterized by IR, NMR UV-Vis, and single-crystal structure analysis $\left[\mathrm{H}_{2} \mathrm{~L}^{1}=(E)-N^{\prime}-((2\right.$-hydroxynaphthalen-1-yl)methylene)furan-2-carbohydrazide, $\mathrm{H}_{2} \mathrm{~L}^{2}=(E)-\mathrm{N}^{\prime}$-(2-hydroxybenzylidene)furan-2-carbohydrazide, $\mathrm{H}_{2} \mathrm{~L}^{3}=(E)-\mathrm{N}^{\prime}$-(5-bromo-2-hydroxybenzylidene)furan2-carbohydrazide, $\mathrm{H}_{2} \mathrm{~L}^{4}=(E)$ - $N^{\prime}$-(2-hydroxy-5-nitrobenzylidene)furan-2-carbohydrazide, $\mathrm{H}_{2} \mathrm{~L}^{5}=(E)$ - $N^{\prime}$ - $(2$-hydroxy-5iodobenzylidene)furan-2-carbohydrazide]. In all 1-3 structures the vanadium atom has a distorted octahedral coordination with the three meridional donor atoms from the Schiff base dianion $\left(\mathrm{L}^{1-3}\right) 2^{-}$and one methoxylato group occupying the sites of the equatorial plane. The oxo group and one methanol molecule occupy the apical sites. In the complexes $\mathbf{1}, \mathbf{2}$, and $\mathbf{3}$ the conformation of 2 -furanyl oxygen atom relative to the carbohydrazide oxygen atom is $s$-anti, $s$-anti/s-syn, and $s$-syn at $293 \mathrm{~K}$, respectively. Cyclic voltammetric experiments of the solution species 1-5 in DMSO revealed a quasi-reversible behavior.

\section{Introduction}

The chemistry of vanadium has received considerable attention due to the discovery that vanadium is an essential element in biological systems [1]. There is continuous interest in the chemistry of vanadium complexes due to its inhibitory capabilities for various enzymes [2], its ability to catalyze oxidation and oxo-transfer reactions [3], and its occurrence in many biological systems [4]. This is particularly related with the discoveries of several medicinal properties of vanadium complexes, that is, insulin mimetic [5-7], antifungal/ antibacterial [8], antitumor [9], anticancer activities [10], and the presence of vanadium in the prosthetic group of certain haloperoxidases and nitrogenases [11]. Structural and functional models for vanadate-dependent haloperoxidases, for vanadium nitrogenases and other biologically active vanadium compounds, have further stimulated vanadium coordination chemistry [12]. Vanadium complexes are also important catalysts for several chemical reactions such as oxidation, epoxidation, and hydroxylation [13]. Schiff base complexes of V (III, IV and V) have been used as catalysis for the electroreduction of $\mathrm{O}_{2}$ to $\mathrm{H}_{2} \mathrm{O}$ in acetonitrile [14]. Because of electrochemical reversibility of V-Schiff base complex derivations, they can be used as electron transfer mediator for modification of different electrode materials, and preparation of chemically modified electrodes with these compounds has been received increasing interest in the field of electroanalysis [15].

On the other hand, hydrazone ligands, a class of Schiff base, derived from the condensation of acid hydrazides (R-CO-NH- $\mathrm{NH}_{2}$ ) with aromatic 2-hydroxy carbonyl compounds are important tridentate $\mathrm{O}, \mathrm{N}, \mathrm{O}$-donor ligands. 

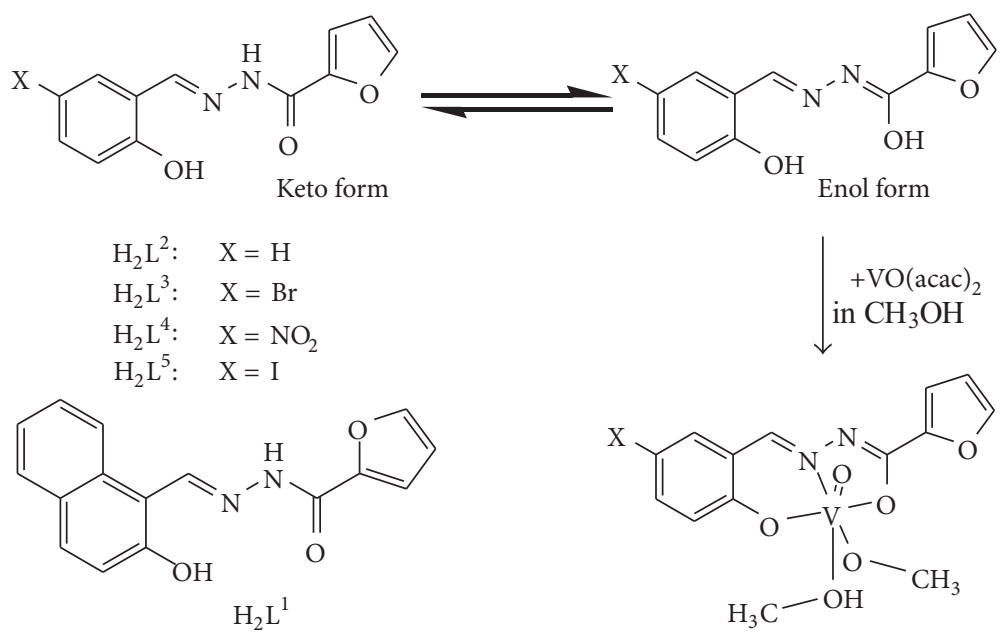

Scheme 1: Tridentate hydrazone Schiff base ligands and their oxovanadium complexes.

The coordination chemistry and biochemistry of aroylhydrazones, $\mathrm{R}-\mathrm{CO}-\mathrm{NH}-\mathrm{N}=\mathrm{CH}-\mathrm{R}^{\prime}$, have attracted increasing interest due to their chelating ability and their pharmacological applications [16]. Hydrazone ligands create environment similar to biological systems by usually making coordination through oxygen and nitrogen atoms [17]. Furthermore, hydrazones have wide spread applications in fields such as coordination chemistry $[18,19]$, analytical chemistry $[20$, 21], bioinorganic chemistry [22-24], and also in magnetic, electronic, nonlinear optically active, and fluorescent [25] compounds.

As part of our research in the study of the coordinating capabilities of aroylhydrazones and their coordination compounds [26-30], here, we report the synthesis, structure, and electrochemistry behavior of monooxovanadium(V) complexes of furancarbohydrazide Schiff bases (Scheme 1).

\section{Experimental}

2.1. Materials and Instrumentations. Vanadyl bis(acetylacetonate), 2-furancarboxylic acid hydrazide, 2-hydroxybenzaldehyde, 5-bromo-2-hydroxybenzaldehyde, 2-hydroxy-5nitrobenzaldehyde, and 2-hydroxy-1-naphthaldehyde were purchased from Merck and used as received. 2-hydroxy-5iodobenzaldehyde was synthesized according to the reported literature procedure [31]. Solvents of the highest grade commercially available (Merck) were used without further purification. IR spectra were recorded in $\mathrm{KBr}$ disks with a Bruker FT-IR spectrophotometer. UV-Vis spectra of solution were recorded on a thermospectronic, Helios Alpha spectrometer. ${ }^{1} \mathrm{H}$ and ${ }^{13} \mathrm{C}$ NMR spectra of ligands and their complexes in DMSO- $\mathrm{d}_{6}$ solution were recorded on a Bruker 250 and $62.9 \mathrm{MHz}$ spectrometer, and chemical shifts are indicated in ppm relative to tetramethylsilane. Voltammetric experiments were performed using an autolab voltammetric analyzer.

\subsection{Synthesis of the Ligands}

2.2.1. General Procedure. All ligands were prepared in a similar manner by refluxing a mixture of 2-furancarboxylic acid hydrazide and $o$-hydroxybenzaldehyde with equivalent molar ratio in $20 \mathrm{~mL}$ methanol. The mixture was refluxed for 2-3 $\mathrm{h}$. The solution was then evaporated on a steam bath to $5 \mathrm{~mL}$ and cooled to room temperature. The obtained solids were separated and filtered off, washed with $5 \mathrm{~mL}$ of cooled methanol, and then dried in air. Completion of the reactions was checked by TLC on silica gel plates.

2.3. Synthesis of (E)-N'-((2-Hydroxynaphthalen-1-yl)methylene)furan-2-carbohydrazide $\left(\mathrm{H}_{2} \mathrm{~L}^{1}\right)$. Yield: $85 \%$. Anal Calc. for $\mathrm{C}_{16} \mathrm{H}_{12} \mathrm{~N}_{2} \mathrm{O}_{3}(280.28 \mathrm{~g} / \mathrm{mol})$ C: $68.56, \mathrm{H}: 4.32, \mathrm{~N}: 9.99 \%$. Found C: $68.50, \mathrm{H}: 4.36, \mathrm{~N}: 10.11 \%$. Selected IR data $\left(\mathrm{cm}^{-1}\right)$ : 1650 (vs, $\mathrm{C}=\mathrm{O}$ ); 1591 (m, C= $\mathrm{N}_{\text {azomethine }}$ ); 951 (m, N-N); 1288, 1321 (s, C-O enolate); 3167 (m, NH); 3612 (m, O-H) $\mathrm{cm}^{-1}$. ${ }^{1} \mathrm{H}$ NMR $\left(250.13 \mathrm{MHz}\right.$; DMSO-d $\left.{ }_{6}\right): \delta 12.68(\mathrm{~s}, 1 \mathrm{H}$, CO-NH-); $12.24(\mathrm{~s}, 1 \mathrm{H},-\mathrm{OH}) ; 9.48(\mathrm{~s}, 1 \mathrm{H}) ; 8.18(\mathrm{~d}, 1 \mathrm{H}$, $J=8.25) ; 7.96(\mathrm{~s}, 1 \mathrm{H}) ; 7.89(\mathrm{~d}, 1 \mathrm{H}, J=9.5 \mathrm{~Hz}) ; 7.85(\mathrm{~d}$, $1 \mathrm{H}, J=9.0 \mathrm{~Hz}) ; 7.56(\mathrm{t}, 1 \mathrm{H}, J=8.0 \mathrm{~Hz}) ; 7.34(\mathrm{~m}, 2 \mathrm{H}) ; 7.19$ $(\mathrm{d}, 1 \mathrm{H}, J=9.0 \mathrm{~Hz}) ; 6.70(\mathrm{t}, 1 \mathrm{H}, J=1.5 \mathrm{~Hz}) \mathrm{ppm} .{ }^{1} \mathrm{H}$ NMR $\left(250.13 \mathrm{MHz}\right.$; DMSO- $\left.\mathrm{d}_{6}+\mathrm{D}_{2} \mathrm{O}\right): \delta=9.36(\mathrm{~s}, 1 \mathrm{H}) ; 8.15(\mathrm{~s}$, $1 \mathrm{H}) ; 7.85(\mathrm{~s}, 3 \mathrm{H}) ; 7.52(\mathrm{~s}, 1 \mathrm{H}) ; 7.30(\mathrm{~s}, 2 \mathrm{H}) ; 7.14(\mathrm{~s}, 1 \mathrm{H}) ; 6.66$ $(\mathrm{s}, 1 \mathrm{H}) \mathrm{ppm} .{ }^{13} \mathrm{C}$ NMR (DMSO-d $\left.\mathrm{d}_{6} ; 62.90 \mathrm{MHz}\right): 109.0,112.7$, $115.9,119.3,121.1,124.0,128.2,128.2,129.4,132.0,133.2$, $146.5,146.6,147.5,154.2$, and $158.4 \mathrm{ppm}$. UV/Vis $\left(\mathrm{CH}_{3} \mathrm{OH}\right.$ solution, $\varepsilon_{\max }$ in $\left.10^{3} \mathrm{dm}^{3} \mathrm{~mol}^{-1} \mathrm{~cm}^{-1}\right): 233$ (44870), 266 (22 980), $314\left(16740^{\mathrm{sh}}\right), 326$ (26 420), $365 \mathrm{~nm}$ (23 240).

\subsection{Synthesis of (E)-N'-(2-Hydroxybenzylidene)furan-}

2-carbohydrazide $\left(\mathrm{H}_{2} \mathrm{~L}^{2}\right)$. Yield: $88 \%$. Anal Calc. for $\mathrm{C}_{12} \mathrm{H}_{9} \mathrm{BrN}_{2} \mathrm{O}_{3}(230.22 \mathrm{~g} / \mathrm{mol}) \mathrm{C}: 62.60, \mathrm{H}: 4.38, \mathrm{~N}: 12.17 \%$. 


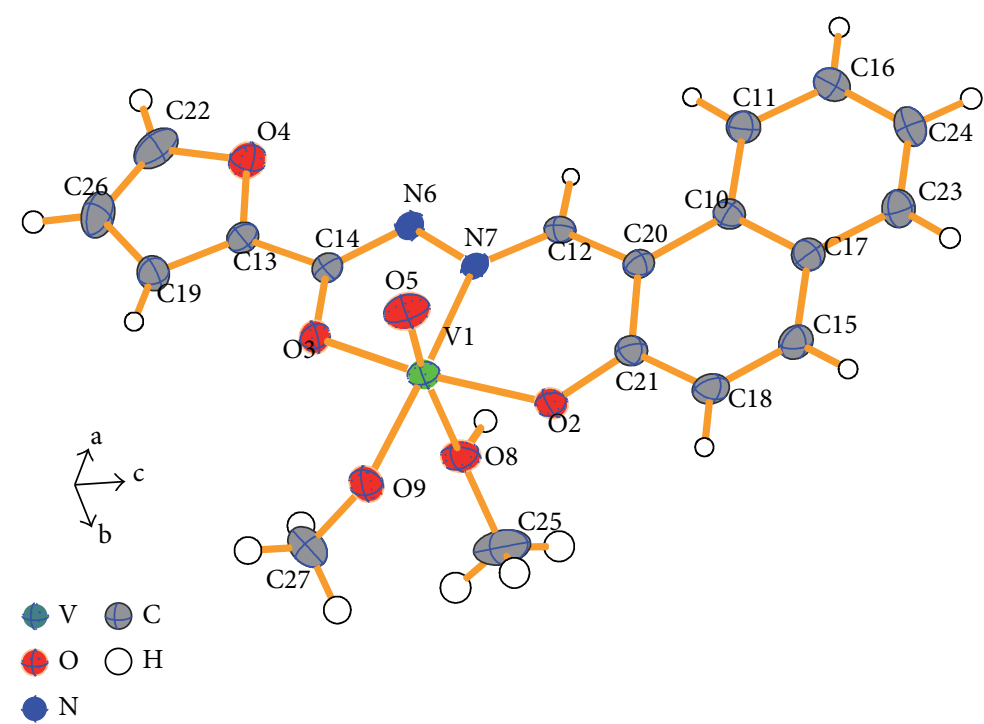

FIgURE 1: Thermal ellipsoid plot of $\left[\mathrm{VO}\left(\mathrm{L}^{1}\right)\left(\mathrm{OCH}_{3}\right)\left(\mathrm{OHCH}_{3}\right)\right](\mathbf{1})$ at 30\% probability level; bond lengths and angles in Table 4 .

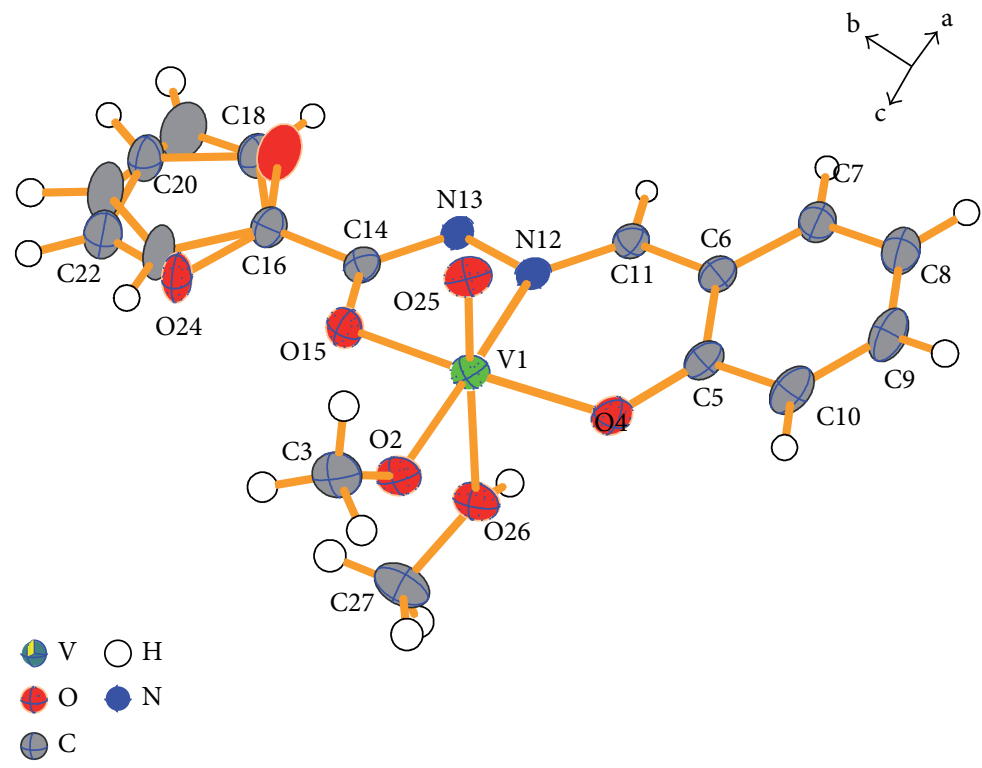

FIgURE 2: Thermal ellipsoid plot of $\left[\mathrm{VO}\left(\mathrm{L}^{2}\right)\left(\mathrm{OCH}_{3}\right)\left(\mathrm{OHCH}_{3}\right)\right](2)$ at $30 \%$ probability level, bond lengths and angles in Table 5 .

Found C: $62.51, \mathrm{H}: 4.34, \mathrm{~N}: 12.22 \%$. Selected IR data $\left(\mathrm{cm}^{-1}\right)$ : 1660 (vs, C=O); 1593 (s, C=N $\mathrm{N}_{\text {azomethine }}$ ); 947 (m, N-N); 1286 (vs, C-O enolate); $3145(\mathrm{~m}, \mathrm{NH}) ; 3621(\mathrm{~m}, \mathrm{O}-\mathrm{H}) \mathrm{cm}^{-1} \cdot{ }^{1} \mathrm{H}$ NMR (250 MHz; DMSO-d $\left.{ }_{6}\right): \delta 12.13$ (s, 1H, CO-NH-); $11.16(\mathrm{~s}, 1 \mathrm{H},-\mathrm{OH}) ; 8.26(\mathrm{~s}, 1 \mathrm{H}) ; 7.92(\mathrm{~s}, 1 \mathrm{H}) ; 7.51(\mathrm{~d}, 1 \mathrm{H}$, $J=7.0 \mathrm{~Hz}) ; 7.27(\mathrm{~m}, 2 \mathrm{H}) ; 6.89(\mathrm{~m}, 2 \mathrm{H}) ; 6.68$ (s, 1H) ppm. ${ }^{1} \mathrm{H}$ NMR $\left(250.13 \mathrm{MHz}\right.$; DMSO-d $\left.\mathrm{d}_{6}+\mathrm{D}_{2} \mathrm{O}\right): \delta=8.52(\mathrm{~s}$, $1 \mathrm{H}) ; 7.78$ (s, 1H); 7.44 (d, 1H, J = 7.25); 7.24 (m, 2H); 6.84 $(\mathrm{m}, 2 \mathrm{H}) ; 6.62(\mathrm{t}, 1 \mathrm{H}, J=1.25) \mathrm{ppm} .{ }^{13} \mathrm{C}$ NMR (DMSO-d $\mathrm{d}_{6}$; $62.90 \mathrm{MHz}$ ): 112.7, 115.7, 116.9, 119.1, 120.0, 129.8, 131.9, $146.45,146.6,148.7,154.5$, and $157.8 \mathrm{ppm}$. UV/Vis $\left(\mathrm{CH}_{3} \mathrm{OH}\right.$ solution, $\varepsilon_{\max }$ in $\left.10^{3} \mathrm{dm}^{3} \mathrm{~mol}^{-1} \mathrm{~cm}^{-1}\right): 239\left(6400^{\mathrm{sh}}\right), 290$ (14080), 300 (15280), $332 \mathrm{~nm}$ (10 980).
2.5. Synthesis of (E)-N'-(5-Bromo-2-hydroxybenzyl-idene) furan-2-carbohydrazide $\left(\mathrm{H}_{2} \mathrm{~L}^{3}\right)$. Yield: 91\%. Anal Calc. for $\mathrm{C}_{12} \mathrm{H}_{9} \mathrm{BrN}_{2} \mathrm{O}_{3}(309.12 \mathrm{~g} / \mathrm{mol}) \mathrm{C}: 46.63, \mathrm{H}: 2.93, \mathrm{~N}$ : 9.06\%. Found C: 46.98, H: 2.89, N: 9.02\%. Selected IR data $\left(\mathrm{cm}^{-1}\right)$ : 1665 (vs, C=O); 1611 (s, C=N azomethine); 956 (m, N-N); 1273, 1306 (s, C-O enolate); 3129 (m, NH); $3397(\mathrm{~m}, \mathrm{O}-\mathrm{H}) \mathrm{cm}^{-1}$. ${ }^{1} \mathrm{H}$ NMR (250 MHz; DMSO-d 6 ): $\delta 12.17(\mathrm{~s}, 1 \mathrm{H}, \mathrm{CO}-\mathrm{NH}-) ; 11.15(\mathrm{~s}, 1 \mathrm{H},-\mathrm{OH}) ; 8.58(\mathrm{~s}$, $1 \mathrm{H}) ; 7.92(\mathrm{~s}, 1 \mathrm{H}) ; 7.74(\mathrm{~s}, 1 \mathrm{H}) ; 7.39(\mathrm{dd}, 1 \mathrm{H}, J=8.75 \mathrm{~Hz}$, $J=2.25 \mathrm{~Hz}) ; 7.30(\mathrm{~s}, 1 \mathrm{H}) ; 6.88(\mathrm{~d}, 1 \mathrm{H}, J=8.5 \mathrm{~Hz}) ; 6.68$ (s, 1H) ppm. ${ }^{1} \mathrm{H}$ NMR $\left(250.13 \mathrm{MHz} ; \mathrm{DMSO}-\mathrm{d}_{6}+\mathrm{D}_{2} \mathrm{O}\right)$ : $\delta=8.50(\mathrm{~s}, 1 \mathrm{H}) ; 7.83(\mathrm{~s}, 1 \mathrm{H}) ; 7.68(\mathrm{~d}, 1 \mathrm{H}, J=2.0) ; 7.84$ $(\mathrm{dd}, 1 \mathrm{H}, J=8.75 \mathrm{~Hz}, J=2.5 \mathrm{~Hz}) ; 7.27(\mathrm{~d}, 1 \mathrm{H}, J=2.5) ; 6.85$ 
TABLE 1: Crystallographic data of 1, 2 and 3.

\begin{tabular}{|c|c|c|c|}
\hline & 1 & 2 & 3 \\
\hline Net formula & $\mathrm{C}_{18} \mathrm{H}_{17} \mathrm{~N}_{2} \mathrm{O}_{6} \mathrm{~V}$ & $\mathrm{C}_{14} \mathrm{H}_{15} \mathrm{~N}_{2} \mathrm{O}_{6} \mathrm{~V}$ & $\mathrm{C}_{14} \mathrm{H}_{14} \mathrm{BrN}_{2} \mathrm{O}_{6} \mathrm{~V}$ \\
\hline$M_{r} / \mathrm{g} \mathrm{mol}^{-1}$ & 408.28 & 358.22 & 437.12 \\
\hline Crystal size/mm & $0.049 \times 0.300 \times 0.455$ & $0.270 \times 0.360 \times 0.450$ & $0.053 \times 0.136 \times 0.565$ \\
\hline$T / \mathrm{K}$ & $293 \mathrm{~K}$ & $293 \mathrm{~K}$ & $293 \mathrm{~K}$ \\
\hline Radiation & $\operatorname{MoK} \alpha$ & $\mathrm{MoK} \alpha$ & $\mathrm{MoK} \alpha$ \\
\hline Crystal system & Monoclinic & monoclinic & monoclinic \\
\hline Space group & $P 2_{1} / c$ & $P 2_{1} / c$ & $P 2_{1} / n$ \\
\hline$\alpha / \AA ̊$ & $7.8995(7)$ & $8.418(2)$ & $10.6835(3)$ \\
\hline$b / \AA$ & $20.3400(10)$ & $16.228(3)$ & $7.8198(3)$ \\
\hline$c / \AA$ & $10.9949(8)$ & $11.902(2)$ & $20.1460(6)$ \\
\hline$\alpha^{\circ} /$ & 90 & 90 & 90 \\
\hline$\beta^{\circ} /$ & $91.577(7)$ & $104.34(2)$ & $104.940(3)$ \\
\hline$\gamma^{\circ} /$ & 90 & 90 & 90 \\
\hline$V / \AA^{3}$ & $1765.95(2)$ & $1575.2(6)$ & 1626.16 \\
\hline$Z$ & 4 & 4 & 4 \\
\hline Calc. density/g cm $\mathrm{cm}^{-3}$ & 1.536 & 1.51 & 1.785 \\
\hline$\mu / \mathrm{mm}^{-1}$ & 0.601 & 0.66 & 3.100 \\
\hline Absorption correction & Analytical & analytical & analytical \\
\hline Refls. measured & 7800 & 7393 & 6829 \\
\hline$R_{\text {int }}$ & 0.035 & 0.031 & 0.038 \\
\hline$\theta$ range & $3.266-29.248$ & $3.3913-28.9660$ & $3.1354-29.2307$ \\
\hline Observed refls. & 2740 & 2732 & 2402 \\
\hline Hydrogen refinement & None & none & none \\
\hline Refls in refinement & 4028 & 3602 & 3736 \\
\hline Parameters & 244 & 245 & 218 \\
\hline Restraints & 0 & 40 & 0 \\
\hline$R\left(F_{\text {obs }}\right)$ & 0.0450 & 0.0519 & 0.0404 \\
\hline$R_{w}\left(F_{2}\right)$ & 0.1399 & 0.1052 & 0.1106 \\
\hline$S$ & 0.9120 & 0.9714 & 0.9785 \\
\hline Shift/error $_{\max }$ & 0.0004097 & 0.0007320 & 0.0003961 \\
\hline
\end{tabular}

$(\mathrm{d}, 1 \mathrm{H}, J=8.75) ; 6.64(\mathrm{t}, 1 \mathrm{H}, J=1.75) \mathrm{ppm} .{ }^{13} \mathrm{C} \mathrm{NMR}$ $\left(\mathrm{DMSO}-\mathrm{d}_{6} ; 62.90 \mathrm{MHz}\right): 110.9,112.6,115.9,119.1,121.8$, $130.6,134.0,146.0,146.7,154.6$, and $156.7 \mathrm{ppm}$. UV/Vis $\left(\mathrm{CH}_{3} \mathrm{OH}\right.$ solution, $\varepsilon_{\max }$ in $\left.10^{3} \mathrm{dm}^{3} \mathrm{~mol}^{-1} \mathrm{~cm}^{-1}\right): 217$ (23020), 246 (16140), 291 (31020), 303 (31460), 340 (18 220).

2.6. Synthesis of (E)-N'-(2-Hydroxy-5-nitrobenzylidene)furan2-carbohydrazide $\left(\mathrm{H}_{2} \mathrm{~L}^{4}\right)$. Yield: 93\%. Anal Calc. for $\mathrm{C}_{12} \mathrm{H}_{9} \mathrm{~N}_{3} \mathrm{O}_{5}(275.22 \mathrm{~g} / \mathrm{mol}) \mathrm{C}: 52.37, \mathrm{H}: 3.30, \mathrm{~N}: 15.27 \%$. Found C: $52.43, \mathrm{H}: 3.35$, N: $15.12 \%$. Selected IR data $\left(\mathrm{cm}^{-1}\right)$ : 1671 (vs, C=O); $1612\left(\mathrm{~m}, \mathrm{C}=\mathrm{N}_{\text {azomethine }}\right) ; 965(\mathrm{~m}, \mathrm{~N}-\mathrm{N})$; 1293, 1344 (vs, C-O enolate); 3274 (m, NH); 3448 (w, O-H) $\mathrm{cm}^{-1}$. ${ }^{1} \mathrm{H}$ NMR $(250.13 \mathrm{MHz} ;$ DMSO-d 6$): \delta 12.22(2 \mathrm{H}$, CO-NH- and -OH); $8.66(\mathrm{~s}, 1 \mathrm{H}) ; 8.48(\mathrm{~d}, 1 \mathrm{H}, J=2 \mathrm{~Hz})$; $8.10(\mathrm{dd}, 1 \mathrm{H}, J=9.25 \mathrm{~Hz}, J=2.5 \mathrm{~Hz}) ; 7.91(\mathrm{~s}, 1 \mathrm{H}) ; 7.31$ $(\mathrm{s}, 1 \mathrm{H}) ; 7.03(\mathrm{~d}, 1 \mathrm{H}, J=9.0 \mathrm{~Hz}) ; 6.67(\mathrm{~d}, 1 \mathrm{H}, J=1.25 \mathrm{~Hz})$ ppm. ${ }^{1} \mathrm{H}$ NMR $\left(250.13 \mathrm{MHz}\right.$; DMSO- $\left.\mathrm{d}_{6}+\mathrm{D}_{2} \mathrm{O}\right): \delta=8.59$ $(\mathrm{s}, 1 \mathrm{H}) ; 8.45(\mathrm{~s}, 1 \mathrm{H}) ; 8.01(\mathrm{~s}, 1 \mathrm{H}) ; 7.82(\mathrm{~s}, 1 \mathrm{H}) ; 7.26(\mathrm{~s}$, $1 \mathrm{H}) ; 7.04(\mathrm{~s}, 1 \mathrm{H}) ; 6.64$ (s, 1H) ppm. ${ }^{13} \mathrm{C}$ NMR (DMSO;
$62.90 \mathrm{MHz}): 112.6,115.9,117.4,120.4,124.0,126.9,140.3$, $144.7,146.6,154.6$, and 162.9. UV/Vis $\left(\mathrm{CH}_{3} \mathrm{OH}\right.$ solution, $\varepsilon_{\max }$ in $\left.10^{3} \mathrm{dm}^{3} \mathrm{~mol}^{-1} \mathrm{~cm}^{-1}\right): 210$ (11680), 295 (28 160), $325 \mathrm{~nm}$ (19 400).

2.7. Synthesis of (E)-N $N^{\prime}-(2-H y d r o x y-5$-iodobenzylidene)furan2-carbohydrazide $\left(\mathrm{H}_{2} \mathrm{~L}^{5}\right)$. Yield: $86 \%$. Anal Calc. for $\mathrm{C}_{12} \mathrm{H}_{9} \mathrm{~N}_{3} \mathrm{O}_{5}$ (275.22 g/mol) C: 40.47, H: 2.55, N: $7.87 \%$. Found C: 40.41, H: 2.61, N: 7.79\%. Selected IR data $\left(\mathrm{cm}^{-1}\right)$ : 1668 (vs, C=O); 1609 (s, C= $\mathrm{N}_{\text {azomethine }}$ ); 952 (m, N-N); 1274, 1351 (vs, C-O enolate); $3262(\mathrm{~m}, \mathrm{NH}) ; 3447$ (w, O-H) $\mathrm{cm}^{-1}$. ${ }^{1} \mathrm{H}$ NMR $\left(250.13 \mathrm{MHz} ; \mathrm{DMSO}-\mathrm{d}^{6}\right): \delta 12.16(\mathrm{~s}, 1 \mathrm{H}$, CO-NH-); 11.15 (s, 1H, -OH); 8.57 (s, 1H); 7.92 (s, 1H); 7.88 $(\mathrm{s}, 1 \mathrm{H}) ; 7.51(\mathrm{~d}, 1 \mathrm{H}, J=8.5 \mathrm{~Hz}) ; 7.30(\mathrm{~s}, 1 \mathrm{H}) ; 7.74(\mathrm{~d}, 1 \mathrm{H}, J=$ $8.75 \mathrm{~Hz} ; 6.67$ (s, 1H) ppm. ${ }^{1} \mathrm{H}$ NMR $\left(250.13 \mathrm{MHz} ; \mathrm{DMSO}_{6}\right.$ $\left.+\mathrm{D}_{2} \mathrm{O}\right): \delta=8.85(\mathrm{~s}, 1 \mathrm{H}) ; 7.83(\mathrm{~s}, 1 \mathrm{H}) ; 7382(\mathrm{~s}, 1 \mathrm{H}) ; 7.24(\mathrm{~s}$, $1 \mathrm{H}) ; 6.72(\mathrm{~d}, 1 \mathrm{H}, J=8.75 \mathrm{~Hz}) ; 6.64(\mathrm{~s}, 1 \mathrm{H}) \mathrm{ppm} .{ }^{13} \mathrm{C} \mathrm{NMR}$ (DMSO; 62.90 MHz): 81.72, 112.61, 115.82, 119.48, 122.35, $136.62,139.76,145.99,146.52,146.63,154.56$, and 157.35. UV/Vis $\left(\mathrm{CH}_{3} \mathrm{OH}\right.$ solution, $\varepsilon_{\max }$ in $\left.10^{3} \mathrm{dm}^{3} \mathrm{~mol}^{-1} \mathrm{~cm}^{-1}\right)$ : 


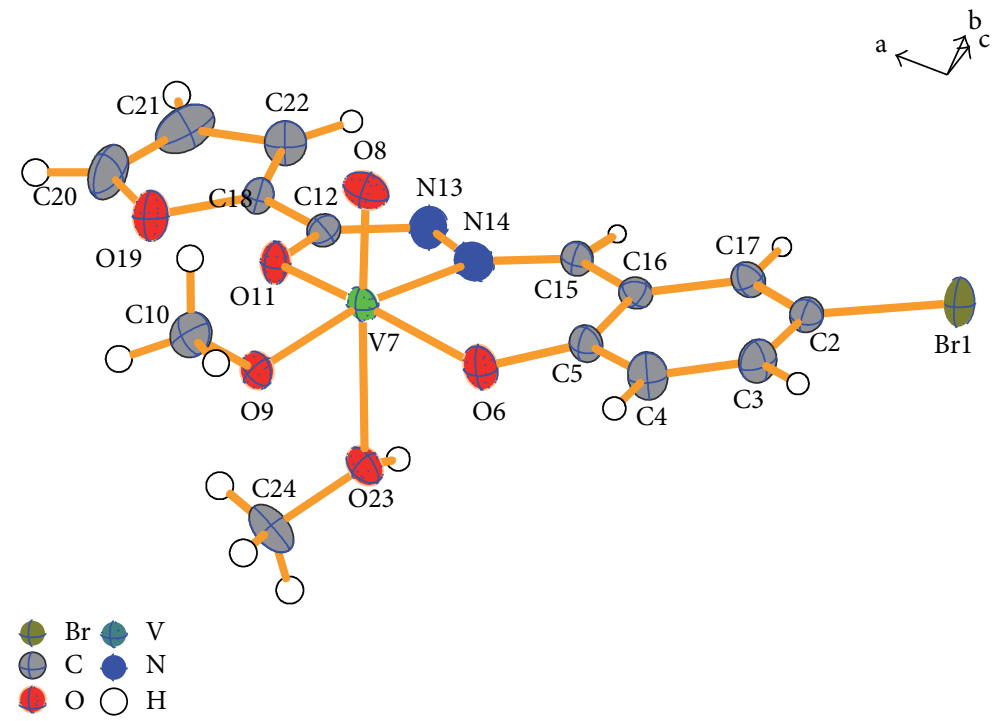

FIgURE 3: Thermal ellipsoid plot of $\left[\mathrm{VO}\left(\mathrm{L}^{3}\right)\left(\mathrm{OCH}_{3}\right)\left(\mathrm{OHCH}_{3}\right)\right](3)$ at $30 \%$ probability level, bond lengths and angles in Table 6.

223 (18 200), 250 (15600), 293 (27 740), 305 (27 680), 344 (14420).

2.8. Synthesis of the Complexes [ $\left.\mathrm{VO}\left(\mathrm{L}^{1-5}\right)\left(\mathrm{OCH}_{3}\right)\left(\mathrm{CH}_{3} \mathrm{OH}\right)\right]$ (1-5). These complexes were synthesized by the same method. General method: the appropriate ligand $\left(\mathrm{H}_{2} \mathrm{~L}^{1}\right.$, $\mathrm{H}_{2} \mathrm{~L}^{2}, \mathrm{H}_{2} \mathrm{~L}^{3}, \mathrm{H}_{2} \mathrm{~L}^{4}$, or $\left.\mathrm{H}_{2} \mathrm{~L}^{5}\right)(1.0 \mathrm{mmol})$ was dissolved in a solution of methanol $(20 \mathrm{~mL})$ then $\mathrm{VO}(\mathrm{acac})_{2} \cdot(0.265 \mathrm{~g}$, $1.0 \mathrm{mmol}$ ) was added and the solution was refluxed for $4 \mathrm{~h}$. After cooling, the resulting solid was filtered off, washed with cooled absolute ethanol, and dried at $100^{\circ} \mathrm{C}$. Single crystals of $\left[\mathrm{VO}\left(\mathrm{L}^{1}\right)\left(\mathrm{OCH}_{3}\right)\left(\mathrm{OHCH}_{3}\right)\right](\mathbf{1}),\left[\mathrm{VO}\left(\mathrm{L}^{2}\right)\left(\mathrm{OCH}_{3}\right)\left(\mathrm{OHCH}_{3}\right)\right]$ (2), and $\left[\mathrm{VO}\left(\mathrm{L}^{3}\right)\left(\mathrm{OCH}_{3}\right)\left(\mathrm{OHCH}_{3}\right)\right](3)$ were prepared by the thermal gradient method.

2.9. Synthesis of $\left[\mathrm{VO}\left(\mathrm{L}^{1}\right)\left(\mathrm{OCH}_{3}\right)\left(\mathrm{OHCH}_{3}\right)\right](\mathbf{1})$. Yield: $82 \%$. Anal Calc. for $\mathrm{C}_{18} \mathrm{H}_{17} \mathrm{~N}_{2} \mathrm{O}_{6} \mathrm{~V}(408.28 \mathrm{~g} / \mathrm{mol}) \mathrm{C}$ : $52.95, \mathrm{H}$ : 4.20, N: 6.86, V: $12.48 \%$. Found C: 53.02, H: 4.13, N: 3.91, V: $12.39 \%$. Selected IR data $\left(\mathrm{cm}^{-1}\right): 3384(\mathrm{~m}) ; 1609$ (vs); 1510 (s); 1330 (s); 1264 (m); 1061 (vs); 973 (vs); 762 (s); 625 (m); $592(\mathrm{~s}) \mathrm{cm}^{-1} .{ }^{1} \mathrm{H}$ NMR $(250.13 \mathrm{MHz}$ DMSO-d 6 ): $\delta 3.15(\mathrm{~s}$, $6 \mathrm{H}) ; 5.27(\mathrm{~s}, 1 \mathrm{H}) ; 6.67(\mathrm{~s}, 1 \mathrm{H}) ; 7.08(\mathrm{~m}, 2 \mathrm{H}) ; 7.45(\mathrm{~s}, 1 \mathrm{H}) ; 7.63$ (s, 1H); $7.91(\mathrm{~s}, 2 \mathrm{H}) ; 8.06(\mathrm{~d}, 1 \mathrm{H}, J=9.25 \mathrm{~Hz}) ; 8.51(\mathrm{~s}, 1 \mathrm{H})$, $9.76(\mathrm{~s}, 1 \mathrm{H}) \mathrm{ppm} .{ }^{13} \mathrm{C}$ NMR (DMSO-d 6 ; $\left.62.90 \mathrm{MHz}\right): 49.0$, $74.4,111.6,112.7,115.3,115.9,119.8,122.1,124.5,128.6$, $128.7,129.4,132.8,135.5,146.4,148.7,162.9$, and $164.7 \mathrm{ppm}$. UV/Vis $\left(\mathrm{CH}_{3} \mathrm{OH}\right.$ solution, $\varepsilon_{\max }$ in $\left.10^{3} \mathrm{dm}^{3} \mathrm{~mol}^{-1} \mathrm{~cm}^{-1}\right): 221$ (39640), $239(38280), 280\left(28280^{\mathrm{b}}\right), 301(26840), 336$ $\left(24880^{\mathrm{sh}}\right), 436 \mathrm{~nm}\left(6920^{\mathrm{sh}}\right)$.

2.10. Synthesis of $\left[\mathrm{VO}\left(\mathrm{L}^{2}\right)\left(\mathrm{OCH}_{3}\right)\left(\mathrm{OHCH}_{3}\right)\right]$ (2). Yield: $75 \%$. Anal Calc. for $\mathrm{C}_{14} \mathrm{H}_{15} \mathrm{~N}_{2} \mathrm{O}_{6} \mathrm{~V}(358.22 \mathrm{~g} / \mathrm{mol}) \mathrm{C}: 46.94, \mathrm{H}$ : 4.22, N: 7.82, V: $14.22 \%$. Found C: $47.04, \mathrm{H}: 4.17, \mathrm{~N}: 7.79$, $\mathrm{V}: 14.25 \%$. Selected IR data $\left(\mathrm{cm}^{-1}\right): 3445(\mathrm{~m}) ; 1612(\mathrm{vs}) ; 1513$ (s); $1351(\mathrm{~m}) ; 1275(\mathrm{~m}) ; 963(\mathrm{~s}) ; 750(\mathrm{~s}) ; 629(\mathrm{~s}) ; 586(\mathrm{~m}) \mathrm{cm}^{-1}$.
${ }^{1} \mathrm{H}$ NMR (250.13 MHz; DMSO-d 6 ): $\delta 3.15$ (s, 6H), $5.26(\mathrm{~s}$, $1 \mathrm{H}) ; 6.64(\mathrm{~s}, 1 \mathrm{H}) ; 6.90(\mathrm{~m}, 2 \mathrm{H}) ; 6.90(\mathrm{~m}, 2 \mathrm{H}) ; 7.11(\mathrm{~s}, 1 \mathrm{H})$; $7.4(\mathrm{~d}, 1 \mathrm{H}, J=5.5 \mathrm{~Hz}) ; 7.66(\mathrm{~s}, 1 \mathrm{H}) ; 7.86(\mathrm{~s}, 1 \mathrm{H}) ; 8.83(\mathrm{~d}, 1 \mathrm{H}$, $J=4 \mathrm{~Hz}$ ) ppm. ${ }^{13} \mathrm{C} \mathrm{NMR}\left(\mathrm{DMSO}-\mathrm{d}_{6} ; 62.90 \mathrm{MHz}\right): 49.0,74.9$, $112.6,115.3,116.6,120.3,120.7,133.3,134.6,146.0,146.4$, $152.4,163.5$, and $163.8 \mathrm{ppm}$. UV/Vis $\left(\mathrm{CH}_{3} \mathrm{OH}\right.$ solution, $\varepsilon_{\max }$ in $\left.10^{3} \mathrm{dm}^{3} \mathrm{~mol}^{-1} \mathrm{~cm}^{-1}\right): 206(34260), 280\left(24880^{\mathrm{sh}}\right), 304$ (26 120), $326\left(26040^{\mathrm{sh}}\right), 410 \mathrm{~nm}\left(5940^{\mathrm{sh}}\right)$.

2.11. Synthesis of $\left[\mathrm{VO}\left(\mathrm{L}^{3}\right)\left(\mathrm{OCH}_{3}\right)\left(\mathrm{OHCH}_{3}\right)\right]$ (3). Yield: $77 \%$. Anal Calc. for $\mathrm{C}_{14} \mathrm{H}_{14} \mathrm{BrN}_{2} \mathrm{O}_{6} \mathrm{~V}$ (437.12 g/mol) C: $38.47, \mathrm{H}$ : 3.23, N: 6.41, V: 11.65\%. Found C: 38.56, H: 3.32, N: 6.34, V: $11.73 \%$. Selected IR data $\left(\mathrm{cm}^{-1}\right): 3557(\mathrm{w}) ; 1609(\mathrm{~s}) ; 1525$ (s); 1341 (s); 1279 (s); 964 (s); 881 (vs); 750 (m); 626 (s); $597(\mathrm{~m}) \mathrm{cm}^{-1}$. ${ }^{1} \mathrm{H}$ NMR $\left(250.13 \mathrm{MHz} ; \mathrm{DMSO}-\mathrm{d}_{6}\right): \delta 3.14$ $(\mathrm{s}, 6 \mathrm{H}) ; 5.30(\mathrm{~s}, 1 \mathrm{H}) ; 6.64(\mathrm{~s}, 1 \mathrm{H}) ; 6.82(\mathrm{~d}, 1 \mathrm{H}, \mathrm{J}=8.5 \mathrm{~Hz})$; $7.11(\mathrm{~s}, 1 \mathrm{H}) ; 7.56(\mathrm{~d}, 1 \mathrm{H}, J=8.75 \mathrm{~Hz}) ; 7.87(\mathrm{~s}, 2 \mathrm{H}) ; 8.78$ (s, $1 \mathrm{H})$ ppm. ${ }^{13} \mathrm{C}$ NMR (DMSO-d 6 ; $62.90 \mathrm{MHz}$ ): 49.0, 75.4, $110.8,112.7,115.67,119.2,122.7,134.8,136.6,145.8,146.6$, $151.3,162.4$, and $164.2 \mathrm{ppm}$. UV/Vis $\left(\mathrm{CH}_{3} \mathrm{OH}\right.$ solution, $\varepsilon_{\max }$ in $\left.10^{3} \mathrm{dm}^{3} \mathrm{~mol}^{-1} \mathrm{~cm}^{-1}\right): 203(27680), 248\left(21100^{\mathrm{sh}}\right), 293$ (22 480), 304 (22 760), 327 (22 180), 412 nm (4200).

2.12. Synthesis of $\left[\mathrm{VO}\left(\mathrm{L}^{4}\right)\left(\mathrm{OCH}_{3}\right)\left(\mathrm{OHCH}_{3}\right)\right]$ (4). Yield: $71 \%$. Anal Calc. for $\mathrm{C}_{14} \mathrm{H}_{14} \mathrm{~N}_{3} \mathrm{O}_{8} \mathrm{~V}$ (403.22 g/mol) C: $41.70, \mathrm{H}$ : 3.50, N: 10.42, V: 12.63\%. Found C: 41.85, H: 3.56, N: 10.51, V: $12.60 \%$. Selected IR data $\left(\mathrm{cm}^{-1}\right): 3415(\mathrm{~m}), 1614$ (vs); 1515 (s); 1339 (vs); 1289 (s); 968 (s); 749 (m); 580 (m) $\mathrm{cm}^{-1} \cdot{ }^{1} \mathrm{H}$ NMR $\left(250.13 \mathrm{MHz} ; \mathrm{DMSO}_{6}\right): \delta 3.14(\mathrm{~s}, 6 \mathrm{H})$; $5.41(\mathrm{~s}, 1 \mathrm{H}) ; 6.68(\mathrm{~s}, 1 \mathrm{H}) ; 7.06(\mathrm{~m}, 2 \mathrm{H}) ; 7.94(\mathrm{~s}, 1 \mathrm{H}) ; 8.15$ (s, 1H); $8.70(\mathrm{~s}, 1 \mathrm{H}) ; 9.00(\mathrm{~s}, 1 \mathrm{H}) \mathrm{ppm} .{ }^{13} \mathrm{C}$ NMR spectrum could not be taken because of the solubility limitations. $\mathrm{UV} / \mathrm{Vis}\left(\mathrm{CH}_{3} \mathrm{OH}\right.$ solution, $\varepsilon_{\max }$ in $\left.10^{3} \mathrm{dm}^{3} \mathrm{~mol}^{-1} \mathrm{~cm}^{-1}\right): 215$ (18 600), $238\left(16060^{\mathrm{sh}}\right), 324$ (33600 very broad) and $429 \mathrm{~nm}$ $\left(2420^{\text {sh }}\right)$. 


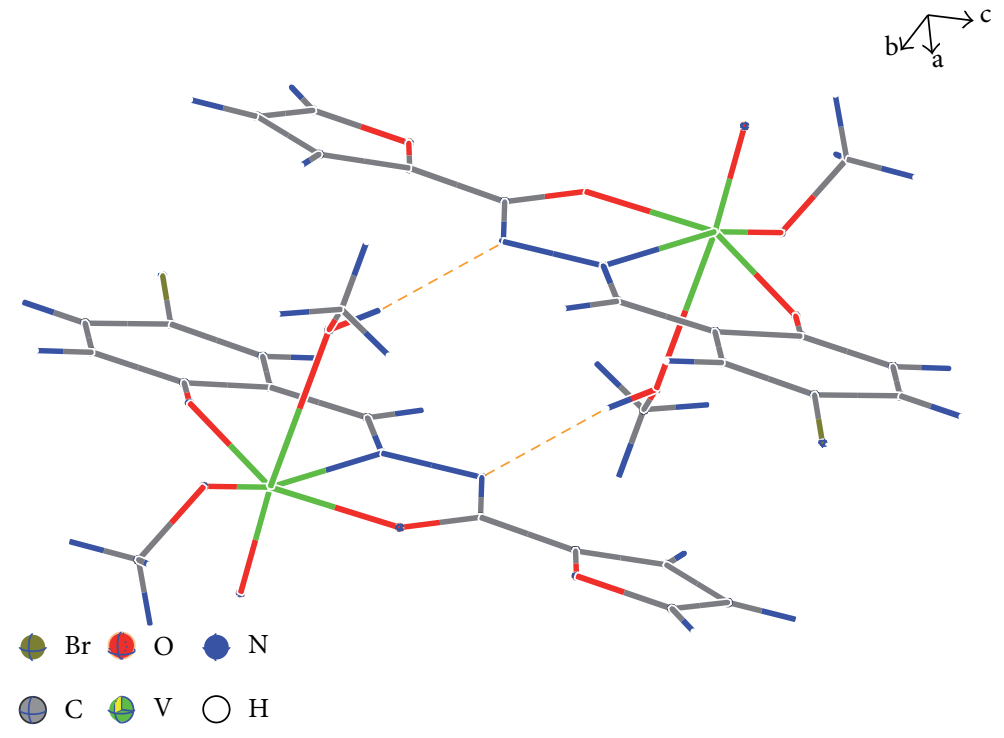

FIgURE 4: The hydrogen-bonded structure of the complex 3.

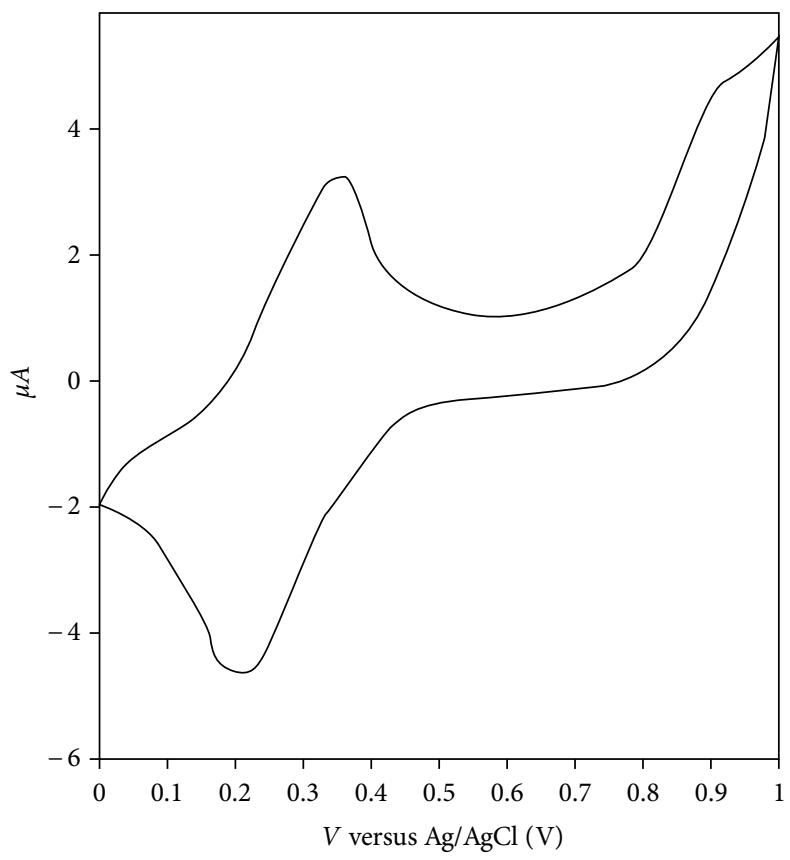

FIGURE 5: Cyclic voltammogram of $10^{-3} \mathrm{~mol} \mathrm{~L}^{-1}$ complex 2 in DMSO and $\mathrm{LiClO}_{4}\left(0.1 \mathrm{~mol} \mathrm{~L}^{-1}\right)$; scan rate $50 \mathrm{mVs}^{-1}$.

2.13. Synthesis of $\left[\mathrm{VO}\left(\mathrm{L}^{5}\right)\left(\mathrm{OCH}_{3}\right)\left(\mathrm{OHCH}_{3}\right)\right]$ (5). Yield: $70 \%$. Anal Calc. for $\mathrm{C}_{14} \mathrm{H}_{14} \mathrm{IN}_{2} \mathrm{O}_{6} \mathrm{~V}(484.12 \mathrm{~g} / \mathrm{mol}) \mathrm{C}: 34.73, \mathrm{H}$ : 2.91, N: 5.79, V: $10.52 \%$. Found C: $34.67, \mathrm{H}: 2.88, \mathrm{~N}: 5.90$, V: $10.68 \%$. Selected IR data $\left(\mathrm{cm}^{-1}\right): 3415$ (s); 1614 (vs); 1516 (s); 1350 (m); 1273 (s); 1051 (vs); 970 (s); 745(m); 626 (s); 591 (s) $\mathrm{cm}^{-1} .{ }^{1} \mathrm{H}$ NMR $\left(250.13 \mathrm{MHz}\right.$; DMSO-d $\left.{ }_{6}\right): \delta 3.15(\mathrm{~s}, 6 \mathrm{H})$; $5.30(\mathrm{~s}, 1 \mathrm{H}) ; 6.64(\mathrm{~s}, 1 \mathrm{H}) ; 6.71(\mathrm{~d}, 1 \mathrm{H}, J=8.5 \mathrm{~Hz}) ; 7.12(\mathrm{~s}, 1 \mathrm{H})$; $7.69(\mathrm{~m} \mathrm{~d}, 1 \mathrm{H}, J=8.0 \mathrm{~Hz}) ; 7.85(\mathrm{~s}, 1 \mathrm{H}) ; 8.00(\mathrm{~s}, 1 \mathrm{H}) ; 8.76$ (s, $1 \mathrm{H}) \mathrm{ppm} .{ }^{13} \mathrm{C}$ NMR (DMSO-d 6 ; $\left.62.90 \mathrm{MHz}\right): 48.8,75.3$, $81.4,112.6,115.6,119.5,123.4,140.8,142.3,145.8,146.4$, $151.1,163.0$, and $164.2 \mathrm{ppm}$. UV/Vis $\left(\mathrm{CH}_{3} \mathrm{OH}\right.$ solution, $\varepsilon_{\max }$ in $\left.10^{3} \mathrm{dm}^{3} \mathrm{~mol}^{-1} \mathrm{~cm}^{-1}\right): 240(21560), 285(21680), 306$ (23040), $334\left(20280^{\mathrm{sh}}\right), 426 \mathrm{~nm}\left(2900^{\mathrm{sh}}\right)$.

2.14. Electrochemical Properties. For cyclic voltammetry studies, a conventional three-electrode system was used with a polished glassy carbon electrode (area $3.14 \mathrm{~mm}^{2}$ ) as working electrode and a platinum wire counter electrode. The reference was an aqueous $\mathrm{Ag} / \mathrm{AgCl}$ saturated electrode, separated from the bulk of the solution by a bridge with solvent and supporting electrolyte. The solutions in the bridge 
TABLE 2: Selected bond lengths $(\AA)$ and angles $\left({ }^{\circ}\right)$ for $\mathbf{1}$.

\begin{tabular}{lc}
\hline Bond lengths & \\
\hline V1-O2 & $1.8480(18)$ \\
V1-O3 & $1.9430(17)$ \\
V1-O5 & $1.585(2)$ \\
V1-N7 & $2.078(2)$ \\
V1-O8 & $2.569(2)$ \\
V1-O9 & $1.7652(18)$ \\
O3-C14(C-O) & $1.307(3)$ \\
O2-C21(C-O) & $1.337(3)$ \\
N6-C14(C=N) & $1.305(3)$ \\
N7-C12(C=N) & $1.297(3)$ \\
N6-N7 & $1.396(3)$ \\
O8-H81 & 0.837 \\
\hline Bond angles & \\
\hline O2-V1-O3 & $152.03(8)$ \\
O2-V1-O5 & $99.87(11)$ \\
O3-V1-O5 & $101.00(10)$ \\
O2-V1-N7 & $84.07(8)$ \\
O3-V1-N7 & $75.18(7)$ \\
O5-V1-N7 & $96.74(9)$ \\
O2-V1-O8 & $78.99(8)$ \\
O3-V1-O8 & $77.77(7)$ \\
O5-V1-O8 & $172.09(9)$ \\
N7-V1-O8 & $75.37(7)$ \\
O2-V1-O9 & $103.01(8)$ \\
O3-V1-O9 & $89.51(8)$ \\
O5-V1-O9 & $104.61(11)$ \\
N7-V1-O9 & $155.81(9)$ \\
O8-V1-O9 & $83.24(8)$ \\
V1-O2-C21 & $133.69(16)$ \\
V1-O3-C14 & $117.39(15)$ \\
V1-N7-N6 & $116.56(14)$ \\
V1-N7-C12 & $127.44(17)$ \\
\hline &
\end{tabular}

were changed periodically to avoid aqueous contamination from entering the cell via the $\mathrm{Ag} / \mathrm{AgCl}$ electrode. The electrolytic medium consisted of $0.1 \mathrm{~mol} / \mathrm{L}$ lithium perchlorate $\left(\mathrm{LiClO}_{4}\right)$ as supporting electrolyte in dimethyl sulfoxide, and all experiments were carried out at room temperature. The solutions were freshly prepared before use and were purged with $\mathrm{N}_{2}$ saturated with solvent for $10 \mathrm{~min}$ prior to taking measurements in order to remove dissolved $\mathrm{O}_{2}$. Voltammograms were recorded in the range from 0.0 to +1.0 V versus Ag/AgCl.

2.15. X-Ray Crystallography Data Collection and Refinement. Dark brown crystals of $\mathbf{1}, \mathbf{2}$, and 3 were investigated by X-ray diffraction at $200 \mathrm{~K}$ on an Oxford-Diffraction Xcalibur Nova E diffractometer equipped with a molybdenum microsource $(\lambda=0.7107 \AA)$. The structures were solved by Direct Methods with SIR97 [32] and refined with full-matrix least-squares techniques on $F^{2}$ with CRYSTALS [33]. The crystal data and refinement parameters are presented in Table 1 . The hydrogen atoms were found in successive Fourier difference
TABLE 3: Selected bond lengths $(\AA)$ and angles $\left({ }^{\circ}\right)$ for 2.

\begin{tabular}{lc}
\hline Bond lengths & \\
\hline V1-O2 & $1.7649(19)$ \\
V1-O4 & $1.848(2)$ \\
V1-N12 & $2.121(2)$ \\
V1-O15 & $1.9726(19)$ \\
V1-O25 & $1.593(2)$ \\
V1-O26 & $2.329(2)$ \\
C14-O15 & $1.292(3)$ \\
O4-C5 & $1.333(3)$ \\
C11-N12 & $1.286(3)$ \\
N13-C14 & $1.310(3)$ \\
N12-N13 & $1.393(3)$ \\
O26-H261 & 0.825 \\
\hline Bond angles & \\
\hline O2-V1-O4 & $102.81(9)$ \\
O2-V1-N12 & $160.64(9)$ \\
O2-V1-O26 & $81.50(8)$ \\
O2-V1-O15 & $93.40(9)$ \\
O2-V1-O25 & $102.97(10)$ \\
O4-V1-O15 & $152.49(8)$ \\
O4-V1-O25 & $99.60(10)$ \\
O4-V1-O26 & $80.65(9)$ \\
N12-V1-O15 & $74.38(7)$ \\
N12-V1-O25 & $93.74(9)$ \\
N12-V1-O26 & $81.60(7)$ \\
O15-V1-O25 & $98.18(10)$ \\
O15-V1-O26 & $79.96(8)$ \\
O25-V1-O26 & $175.30(8)$ \\
V1-O2-C3 & $131.02(19)$ \\
O4-V1-N12 & $83.65(8)$ \\
\hline &
\end{tabular}

analysis. All nonhydrogen atoms were refined anisotropically. Hydrogen atoms were first refined with restraints on the bond lengths and angles $(\mathrm{C}-\mathrm{H}$ in the range $0.93-0.98 \AA$ and $\mathrm{O}-\mathrm{H}=$ $0.82 \AA$ ) and $\mathrm{U}_{\text {iso }}$ (in the range 1.2-1.5 times $\mathrm{U}_{\text {eq }}$ of the parent atom), after which they were refined with riding constraints. The molecular structure plots were drawn with ORTEPIII [34-36].

\section{Results and Discussion}

The reaction of 2-furancarboxylic acid hydrazide with several aromatic $o$-hydroxy aldehydes with different substituents in methanol gave the desired tridentate Schiff base ligands in excellent yields and purity. Oxovanadium(V) complexes with tridentate hydrazone Schiff base ligands were prepared by treating a methanolic solution of the appropriate ligand with equimolar amount of $\mathrm{VO}(\mathrm{acac})_{2}$ (Scheme 1$)$.

3.1. Description of the Structures (1, 2, and 3). In order to define the coordination sphere conclusively, a singlecrystal X-ray diffraction study was made. A list of some crystallographic data of $\mathbf{1}, \mathbf{2}$, and 3 is given in Table 1. An ORTEP diagram with the atom numbering scheme of the $\mathbf{1 , 2}$, and 3 is shown in Figures 1, 2, and 3 and selected bond lengths 
TABLE 4: Selected bond lengths $(\AA)$ and angles $\left(^{\circ}\right)$ for 3.

\begin{tabular}{lc}
\hline Bond lengths & \\
\hline V7-O6 & $1.847(3)$ \\
V7-O8 & $1.584(4)$ \\
V7-O9 & $1.767(3)$ \\
V7-O11 & $1.957(3)$ \\
V7-N14 & $2.130(3)$ \\
V7-O23 & $2.372(4)$ \\
O11-C12 (C-O) & $1.287(5)$ \\
C5-O6 (C-O) & $1.341(5)$ \\
C12-N13 (C=N) & $1.314(5)$ \\
N14-C15 (C=N) & $1.289(5)$ \\
N13-N14 & $1.399(5)$ \\
\hline Bond angles & \\
\hline O6-V7-O8 & $99.77(19)$ \\
O6-V7-O9 & $100.85(14)$ \\
O8-V7-O9 & $103.06(17)$ \\
O6-V7-O11 & $152.96(15)$ \\
O8-V7-O11 & $97.74(17)$ \\
O9-V7-O11 & $95.04(14)$ \\
O6-V7-N14 & $83.93(13)$ \\
O8-V7-N14 & $94.85(16)$ \\
O9-V7-N14 & $160.28(16)$ \\
O8-V7-O23 & $174.55(15)$ \\
O9-V7-O23 & $81.83(14)$ \\
O11-V7-O23 & $79.28(14)$ \\
N14-V7-O23 & $79.97(13)$ \\
O6-V7-O23 & $81.48(15)$ \\
V7-N14-N13 & $115.8(2)$ \\
V7-N14-C15 & $127.4(3)$ \\
V7-O11-C12 & $118.6(3)$ \\
O11-V7-N14 &
\end{tabular}

and angles are given in Tables 2, 3, and 4, respectively. In 1, $\mathbf{2}$, and $\mathbf{3}$, the vanadium atom has a six-coordinated structure as a $\mathrm{VO}_{5} \mathrm{~N}$ with nitrogen and two oxygen atoms provided by the Schiff base ligand and three oxygen atoms from methoxy, methanol, and oxo ligands. An axial position is occupied by the oxygen atom from methanol, and another axial position is occupied by the oxygen atom from the oxo ligand.

In these compounds the Schiff base ligands form a six-membered and a five-membered chelate ring with bite angles of about $84^{\circ}\left(\mathrm{O}_{\text {phenolat }}-\mathrm{V}-\mathrm{N}\right)$ and $74^{\circ}\left(\mathrm{N}-\mathrm{V}-\mathrm{O}_{\text {enolat }}\right)$, respectively. This angles are the same with previously reported naphthohydrazone oxovanadium complexes [29]. The double deprotonated form of the $\mathrm{N}$-arylidene fouranohydrazide ligands is consistent with the observed $\mathrm{O}-\mathrm{C}_{\text {carbonyl }}$ and $\mathrm{N}=\mathrm{C}_{\text {carbonyl }}$ bond lengths of 1.29 and $1.31 \AA$, respectively in 1,2 , and 3 . This is in agreement with the reported complexes containing the enolate form of $\mathrm{N}$-arylidene hydrazone ligands $[29,30,37]$, whereas the $\mathrm{C}=\mathrm{O}$ bond is considerably short for reported complexes with the coordinated keto form of the $\mathrm{N}$-arylidene benzohydrazide system [28, 38]. In furancarbohydrazide ligands the $\mathrm{C}-\mathrm{N}$ and $\mathrm{C}=\mathrm{O}$ bond
TABLE 5: Hydrogen bonding interactions for $\mathbf{1 , 2}$ and $\mathbf{3 .}$

\begin{tabular}{|c|c|c|c|c|}
\hline $\mathrm{D}-\mathrm{H} \cdots \mathrm{A}$ & $\begin{array}{c}\text { D-H } \\
(\AA)\end{array}$ & $\begin{array}{c}\mathrm{H} \cdots \mathrm{A} \\
(\AA)\end{array}$ & $\begin{array}{c}\mathrm{D} \cdots \mathrm{A} \\
(\AA)\end{array}$ & $\begin{array}{c}\text { D-H } \cdots A \\
(\mathrm{deg})\end{array}$ \\
\hline \multicolumn{5}{|l|}{ Complex 1} \\
\hline O $8-\mathrm{H} 81 \cdots \mathrm{N} 6$ & 0.84 & 2.01 & $2.842(4)$ & 176 \\
\hline \multicolumn{5}{|l|}{ Complex 2} \\
\hline $\mathrm{O} 26-\mathrm{H} 261 \cdots \mathrm{N} 13$ & 0.82 & 2.01 & $2.817(8)$ & 165 \\
\hline \multicolumn{5}{|l|}{ Complex 3} \\
\hline $\mathrm{O} 23-\mathrm{H} 231 \cdots \mathrm{N} 13$ & 0.82 & 2.06 & $2.876(7)$ & 171 \\
\hline
\end{tabular}

lengths are about $1.35 \AA$ and $1.23 \AA$, respectively [39-41]. The corresponding bond lengths in complex 1-3 are about $1.31 \AA$ and $1.29 \AA$, respectively. Comparison of these bond lengths indicates the shortening of the $\mathrm{C}-\mathrm{N}$ bond length and lengthening of the $\mathrm{C}=\mathrm{O}$ bond due to coordination in enol form. The vanadium to oxygen bond lengths follows the order $\mathrm{V}$-oxo oxygen $<\mathrm{V}$-methoxy oxygen $<\mathrm{V}$-phenolate oxygen $<\mathrm{V}$-enolate oxygen $<\mathrm{V}$-methanol oxygen. The oxovanadium (V) complexes under consideration crystallize in the monoclinic crystal system. In $\mathbf{1}$ and $\mathbf{2}$ the space group is $P 2_{1} / c$ but in 3 , the space group is $P 2_{1} / n$.

In the complexes $\mathbf{1}, \mathbf{2}$, and 3 the conformation of 2 furanyl oxygen atom relative to the carbohydrazide oxygen atom is $s$-anti, $s$-anti/s-syn, and $s$-syn at $293 \mathrm{~K}$, respectively. These findings suggest the presence of low barrier energy for rotation of the 2-furanyl group around the $\mathrm{C}-\mathrm{C}$ bond between 2-furanyl and the carbohydrazide groups at room temperature. This rotation plausibly prevents 2 -furanyl oxygen atom involvement in the coordination to the vanadium center of the adjacent molecule. In addition, the oxygen atom in furan is too poor for a donor to take part in metal binding. From steric consideration also, the furan group is not properly positioned to be involved in metal binding.

Hydrogen bonding is a common feature for vanadium(IV) and vanadium(V) compounds in the solid state, if appropriate hydrogen bonding donors are present [42, 43]. The type of complexes described in this work contains two major functionalities which can participate in intermolecular hydrogen bond interactions. These are the $\mathrm{N}$ atom of the hydrazine fragment of the tridentate ligand and the $\mathrm{O}-\mathrm{H}$ group of the metal coordinated methanol. In these complexes two molecules of complex are connected together by strong intermolecular $\mathrm{O}_{\text {(methanol) }}-\mathrm{H} \cdots \mathrm{N}_{\text {(amide) }}$ hydrogen bonds and create a pseudodimer as depicted in Figure 4. This strong intermolecular hydrogen bond stabilizes the crystal structure of 1,2 , and 3 . These pseudodimers make a chain along $c$ diagonal by intermolecular hydrogen bonds. Parameters of hydrogen bonding geometry are given in Table 5 .

\subsection{Spectroscopic Studies}

3.2.1. NMR Spectra of the Ligands and Complexes. ${ }^{1} \mathrm{H}$ and ${ }^{13} \mathrm{C}$ NMR spectral data of the ligands in DMSO- $\mathrm{d}_{6}$ confirmed the proposed structure of the ligands (Scheme 1). The principal peaks of the ${ }^{1} \mathrm{H}$ NMR spectra of ligands $\mathrm{H}_{2} \mathrm{~L}^{1}-\mathrm{H}_{2} \mathrm{~L}^{5}$ 
TABLe 6: ${ }^{1} \mathrm{H}$ NMR of ligands $\left(\mathrm{H}_{2} \mathrm{~L}^{1}-\mathrm{H}_{2} \mathrm{~L}^{5}\right)$ and complexes (1-5).

\begin{tabular}{|c|c|c|c|c|c|c|}
\hline Compound & Solvent & $-\mathrm{CH}=\mathrm{N}-$ (azomethine) & $-\mathrm{NH}-$ & $-\mathrm{OH}_{(\text {phenol })}$ & $-\mathrm{OH}_{(\text {methanol })}$ & $-\mathrm{CH}_{3}$ \\
\hline $\mathrm{H}_{2} \mathrm{~L}^{1}$ & $\left(\mathrm{CD}_{3}\right)_{2} \mathrm{SO}$ & 8.66 & 12.30 & 11.31 & - & - \\
\hline $\mathrm{H}_{2} \mathrm{~L}^{1}$ & $\left(\mathrm{CD}_{3}\right)_{2} \mathrm{SO}+\mathrm{D}_{2} \mathrm{O}$ & 8.58 & - & - & - & - \\
\hline Complex 1 & $\left(\mathrm{CD}_{3}\right)_{2} \mathrm{SO}$ & 9.76 & - & - & 5.27 & 3.15 \\
\hline $\mathrm{H}_{2} \mathrm{~L}^{2}$ & $\left(\mathrm{CD}_{3}\right)_{2} \mathrm{SO}$ & 8.61 & 12.33 & 11.13 & - & - \\
\hline $\mathrm{H}_{2} \mathrm{~L}^{2}$ & $\left(\mathrm{CD}_{3}\right)_{2} \mathrm{SO}+\mathrm{D}_{2} \mathrm{O}$ & 8.57 & - & - & - & - \\
\hline Complex 2 & $\left(\mathrm{CD}_{3}\right)_{2} \mathrm{SO}$ & 8.83 & - & - & 5.27 & 3.15 \\
\hline $\mathrm{H}_{2} \mathrm{~L}^{3}$ & $\left(\mathrm{CD}_{3}\right)_{2} \mathrm{SO}$ & 8.67 & 12.25 & 10.69 & - & - \\
\hline $\mathrm{H}_{2} \mathrm{~L}^{3}$ & $\left(\mathrm{CD}_{3}\right)_{2} \mathrm{SO}+\mathrm{D}_{2} \mathrm{O}$ & 8.58 & - & - & - & - \\
\hline Complex 3 & $\left(\mathrm{CD}_{3}\right)_{2} \mathrm{SO}$ & 8.78 & - & - & 5.30 & 3.14 \\
\hline $\mathrm{H}_{2} \mathrm{~L}^{4}$ & $\left(\mathrm{CD}_{3}\right)_{2} \mathrm{SO}$ & 8.66 & 12.22 & 12.22 & - & - \\
\hline $\mathrm{H}_{2} \mathrm{~L}^{4}$ & $\left(\mathrm{CD}_{3}\right)_{2} \mathrm{SO}+\mathrm{D}_{2} \mathrm{O}$ & 8.59 & - & - & - & - \\
\hline Complex 4 & $\left(\mathrm{CD}_{3}\right)_{2} \mathrm{SO}$ & 8.99 & - & - & 5.41 & 3.14 \\
\hline $\mathrm{H}_{2} \mathrm{~L}^{5}$ & $\left(\mathrm{CD}_{3}\right)_{2} \mathrm{SO}$ & 8.57 & 12.16 & 11.15 & - & - \\
\hline $\mathrm{H}_{2} \mathrm{~L}^{5}$ & $\left(\mathrm{CD}_{3}\right)_{2} \mathrm{SO}+\mathrm{D}_{2} \mathrm{O}$ & 8.85 & - & - & - & - \\
\hline Complex 5 & $\left(\mathrm{CD}_{3}\right)_{2} \mathrm{SO}$ & 8.76 & - & - & 5.30 & 3.15 \\
\hline
\end{tabular}

TABLE 7: Electrochemical data of the complexes 1-5 in DMSO solution at 298 K.

\begin{tabular}{|c|c|c|c|c|c|}
\hline Compound & $E_{\mathrm{p} . \mathrm{a}}(\mathrm{V})$ & $I_{\text {p.a }}$ & $E_{\text {p.c. }}(\mathrm{V})$ & $I_{\mathrm{p} . \mathrm{c}}$ & $\Delta E_{P}(\mathrm{~V})$ \\
\hline 1 & 0.421 & $3.04 \times 10^{-6}$ & 0.273 & $-4.46 \times 10^{-6}$ & 0.148 \\
\hline 2 & 0.349 & $3.28 \times 10^{-6}$ & 0.211 & $-4.61 \times 10^{-6}$ & 0.138 \\
\hline 3 & 0.454 & $2.94 \times 10^{-6}$ & 0.298 & $-4.39 \times 10^{-6}$ & 0.156 \\
\hline 4 & 0.498 & $2.85 \times 10^{-6}$ & 0.313 & $-4.31 \times 10^{-6}$ & 0.185 \\
\hline 5 & 0.430 & $3.01 \times 10^{-6}$ & 0.277 & $-4.37 \times 10^{-6}$ & 0.153 \\
\hline
\end{tabular}

are listed in Table 6 . The signal at $\delta 12.12-12.33$ in the spectra of $\mathrm{H}_{2} \mathrm{~L}^{1}-\mathrm{H}_{2} \mathrm{~L}^{5}$ is assigned to the common $\mathrm{NH}$-group, concomitant with the observation of a rapid loss of these signals when $\mathrm{D}_{2} \mathrm{O}$ is added to the solution. Also the signals between $\delta 10.62-12.22$ in the spectra of $\mathrm{H}_{2} \mathrm{~L}^{1}-\mathrm{H}_{2} \mathrm{~L}^{5}$ are lost upon addition of $\mathrm{D}_{2} \mathrm{O}$ to the solution. Hence, this signal is assigned to the phenolic $\mathrm{OH}$ group. The resonances between $\delta 8.57-8.67$ are assigned to the azomethine $(-\mathrm{CH}=\mathrm{N}-)$ in the spectra of $\mathrm{H}_{2} \mathrm{~L}^{1}-\mathrm{H}_{2} \mathrm{~L}^{5}$. In all ligands other aromatic protons appear between $\delta 6.60-7.85$. The chemical shifts for these complexes are comparable and very close to each other. On complexation the absence of $\mathrm{N}-\mathrm{H}$ and $\mathrm{O}-\mathrm{H}$ peaks of the ligands shows coordination of $\mathrm{H}_{2} \mathrm{~L}^{1}-\mathrm{H}_{2} \mathrm{~L}^{5}$ as dianionic ligands in enol form (Scheme 1). In these ligands, a triplet peak and two doublet peaks should have been observed around $\delta 6.5-7.5 \mathrm{ppm}$ for furan's hydrogens but because of the low coupling constant (ca. $2 \mathrm{~Hz}$.) [44] and also not using a powerful instrument, only a singlet peak is observed for these hydrogens; however, in three cases $\left(\mathrm{H}_{2} \mathrm{~L}^{1}, \mathrm{H}_{2} \mathrm{~L}^{2}\right.$ and $\left.\mathrm{H}_{2} \mathrm{~L}^{4}\right)$ this splitting could be observed. We have also recorded ${ }^{13} \mathrm{C}$ NMR of ligands and their complexes to provide diagnostic tools for the elucidation of the structures. Assignments of the peaks are similar and are based on the chemical shift and intensity patterns. $\Delta \delta$ observed for carbon atoms in the vicinity of the phenolate, enolate, and azomethine groups suggests their involvement in coordination. Two new signals in complexes appear at $\delta$ about 49 and $75 \mathrm{ppm}$; these signals correspond to methanol and methoxy carbon atoms, respectively.

3.2.2. Infrared Spectra. A list of the important vibrational frequencies (IR spectra) of the free ligands $\left(\mathrm{H}_{2} \mathrm{~L}^{1}-\mathrm{H}_{2} \mathrm{~L}^{5}\right)$ and their oxovanadium complexes, which are useful for determining the mode of coordination of the ligands, are given in the experimental part. A comparison of the spectra of the complexes with the ligands provides evidence for the coordination mode of the ligands in the complexes. Hence significant frequencies are selected by comparing the IR spectra of the ligands with those of oxovanadium complexes. All hydrazone Schiff base ligands $\left(\mathrm{H}_{2} \mathrm{~L}^{1}-\mathrm{H}_{2} \mathrm{~L}^{5}\right)$ exhibit a broad band around $3167-3270 \mathrm{~cm}^{-1}$ due to $\mathrm{NH}$-vibrations. Also in IR spectra of all the ligands very strong band appears around $1650-1680 \mathrm{~cm}^{-1}$ due to $\mathrm{C}=\mathrm{O}$-vibration. In addition a broad band is centered at $3400-3600 \mathrm{~cm}^{-1}$ in $\mathrm{H}_{2} \mathrm{~L}^{1}-\mathrm{H}_{2} \mathrm{~L}^{5}$ due to the $\mathrm{O}-\mathrm{H}$ of the phenol, probably involved in intramolecular hydrogen bonding. The infrared spectra of complexes display IR absorption band around $1610 \mathrm{~cm}^{-1}$ which can be assigned to the $\mathrm{C}=\mathrm{N}$ stretching frequency of the coordinated hydrazone ligand, whereas for the free ligands the same band are observed around $1600 \mathrm{~cm}^{-1}$. Strong $\mathrm{C}=\mathrm{N}$ stretch (around $1600 \mathrm{~cm}^{-1}$ ) indicates the $\mathrm{C}=\mathrm{N}$ group of the coordinated Schiff 
base ligands $[45,46]$. On complexation the absence of $\mathrm{N}-\mathrm{H}$ and $\mathrm{C}=\mathrm{O}$ bands and red shifts in azomethine $(-\mathrm{C}=\mathrm{N}-)$ band [47] of the ligands shows coordination of $\mathrm{H}_{2} \mathrm{~L}^{1}-\mathrm{H}_{2} \mathrm{~L}^{5}$ as three dentate dianionic ligands in enol form (Scheme 1). In all complexes very broad bond around $3380-3440 \mathrm{~cm}^{-1}$ expresses presentce of $-\mathrm{OH}$ and coordination of methanol to the vanadium. The band at $963-972 \mathrm{~cm}^{-1}$ is assigned to $v(\mathrm{~V}=\mathrm{O})$; this band is observed as a new peak for the complexes and is not present in the spectra of the free ligands. Similarity of the IR spectra of the complexes shows the similarity of their structures.

3.2.3. Electronic Spectra. These complexes are shiny dark brown in solid state, but their methanol solutions are brown in color. These solutions have been used to record the electronic spectra. For the oxovanadium(V) compounds, no $\mathrm{d}$ - $\mathrm{d}$ bands are expected because they have a $3 \mathrm{~d}^{0}$ configuration and there are no $\mathrm{d}$ electrons [12]. The hydrazone ligands have bands in the range $209-290$ and $300-342 \mathrm{~nm}$. Based on their extinction coefficients these are assigned as due to $\pi \rightarrow \pi^{*}$ and $\mathrm{n} \rightarrow \pi^{*}$ transitions, respectively $[20,48,49]$. The UV-Vis spectrum of $\mathrm{H}_{2} \mathrm{~L}^{3}, \mathrm{H}_{2} \mathrm{~L}^{4}$, and $\mathrm{H}_{2} \mathrm{~L}^{5}$ which they have electron-withdrawing group (e.g., $\mathrm{Br}, \mathrm{I}$, and $\mathrm{NO}_{2}{ }^{-}$) at the para position with respect to phenolic $\mathrm{OH}$ group in the aryloxy ring is very closet to each other and they have very similar shape (they have two $\lambda_{\max }$ in around 290 and $305 \mathrm{~nm}$ ). The Oxovanadium(V) complexes have bands in the range $205-220$ and $324-336 \mathrm{~nm}$. These bands are assigned as due to intraligand transitions. All bands shift in complexes indicating the coordination of ligands to the metal ions $[50,51]$. The shoulder appeared at about $280 \mathrm{~nm}$ for 1 5 corresponds to LMCT band of $\mathrm{V}=\mathrm{O}$ which is appeared at $274 \mathrm{~nm}$ for $\left[\mathrm{VO}(\mathrm{acac})_{2}\right]$. The lowest energy transition lying around $420 \mathrm{~nm}$ is assigned to LMCT transition of the type $\mathrm{O}_{\text {(phenolic) }} \rightarrow \mathrm{V}^{5+}$ [52-55]. Electronic spectra for these complexes in $\mathrm{MeOH}$ solutions are akin similar electronic structure in solution of these compounds.

3.2.4. Electrochemistry. The electrochemical behaviors of the complexes were studied by cyclic voltammetry techniques in the range of 0.00 to $+1.0 \mathrm{~V}$ at a scan rate of $50 \mathrm{mV} / \mathrm{s}$ in DMSO on a glassy carbon electrode (GCE) and $\mathrm{Ag} / \mathrm{AgC1}$ reference electrode using lithium perchlorate $\left(\mathrm{LiClO}_{4}\right)$ as the supporting electrolyte. All the complexes exhibit a quasireversible reduction peak due to $\mathrm{VO}^{3+} / \mathrm{VO}^{2+}$ couple. Cyclic voltammetry data for these complexes are collected in Table 7 and the Figure 5 displays a representative cyclic voltammogram of 2 . The effect of the electronic nature of the substituent present on the salicylidene fragment of tridentate ligand is clearly reflected on the trend of the $E_{1 / 2}$ values for this reduction. For the strong electron withdrawing substituent $\left(\mathrm{Y}=\mathrm{NO}_{2}\right.$, complex 4) the reduction of the metal centre occurs at the highest potential while for the least (or without any) electron withdrawing substituent $(\mathrm{X}=\mathrm{H}$, complex 2) it occurs at the lowest potential. Also for complex 4 the oxidation of the metal centre occurs at the lowest potential, while for complex $\mathbf{2}$ it occurs at the highest potential.

\section{Conclusion}

This work revealed that coordination complexes of $\mathrm{V}^{5+}$ and tridentate hydrazone Schiff base ligands obtained from the reaction of 2-furancarboxylic acid hydrazide and aromatic $o$-hydroxyaldehydes derivatives afford a new class of $\mathrm{V}^{5+}$ complexes. Five monooxovanadium $(\mathrm{V})$ complexes of tridentate Schiff base ligands were synthesized and characterized by spectroscopic methods and single crystal X-ray analysis. The crystal structures of 1-3 suggest the presence of lowbarrier energy for rotation of the 2-furanyl group around the $\mathrm{C}-\mathrm{C}$ bond between 2-furanyl and the carbohydrazide groups at room temperature. Electrochemical studies by cyclic voltammetry technique indicated that these complexes are quasi-reversible electroactive.

\section{Acknowledgments}

The authors are grateful to the University of Zanjan, the Faculty of Chemistry, Université Claude Bernard Lyon1, and the University of Tabriz for financial support of this study.

\section{References}

[1] M. R. Maurya, S. Agarwal, M. Abid et al., "Synthesis, characterisation, reactivity and in vitro antiamoebic activity of hydrazone based oxovanadium(iv), oxovanadium(v) and -bis(oxo) bisoxovanadium(v) complexes," Dalton Transactions, no. 7, pp. 937-947, 2006.

[2] A. Messerschmidt and R. Wever, "X-ray structure of a vanadium-containing enzyme: chloroperoxidase from the fungus Curvularia inaequalis," Proceedings of the National Academy of Sciences of the United States of America, vol. 93, no. 1, pp. 392-396, 1996.

[3] T. Hirao, "Vanadium in modern organic synthesis," Chemical Reviews, vol. 97, no. 8, pp. 2707-2724, 1997.

[4] F. H. Nelsen, "Vanadium in mammalian physiology and nutrition," in Metal Ions in Biological Systems: Vanadium and Its Role in Life, H. Sigel and A. Siegel, Eds., vol. 31, pp. 543-573, Marcel Dekker, New York, NY, USA, 1995.

[5] D. Rehder, J. C. Pessoa, and C. F. Geraldes, "In vitro study of the insulin-mimetic behaviour of vanadium(IV, V) coordination compounds," Journal of Biological Inorganic Chemistry, vol. 7, no. 6, article 675, 2002.

[6] H. Sakurai, Y. Kojima, Y. Yoshikawa, K. Kawabe, and H. Yasui, "Antidiabetic vanadium(IV) and zinc(II) complexes," Coordination Chemistry Reviews, vol. 226, no. 1-2, pp. 187-198, 2002.

[7] H. Sakurai, K. Tsuchiya, M. Nukatsuka et al., "Insulin-mimetic action of vanadyl complexes," Journal of Clinical Biochemistry and Nutrition, vol. 8, no. 3, pp. 193-200, 1990.

[8] M. J. M. Campbell, "Transition metal complexes of thiosemicarbazide and thiosemicarbazones," Coordination Chemistry Reviews, vol. 15, no. 2-3, pp. 279-319, 1975.

[9] A. Butler and J. V. Walker, "Marine haloperoxidases," Chemical Reviews, vol. 93, no. 5, pp. 1937-1944, 1993.

[10] R. Liasko, T. A. Kabanos, S. Karkabounas et al., "Beneficial effects of a vanadium complex with cysteine, administered at low doses on benzo $(\alpha)$ pyrene-induced leiomyosarcomas in wistar rats," Anticancer Research, vol. 18, no. 5 A, pp. 3609-3613, 1998. 
[11] I. Lippold, K. Vlay, H. Gorls, and W. Plass, "Cyclodextrin inclusion compounds of vanadium complexes: structural characterization and catalytic sulfoxidation," Journal of Inorganic Biochemistry, vol. 103, no. 4, pp. 480-486, 2009.

[12] R. C. Maurya and S. Rajput, "Neutral dioxovanadium(V) complexes of biomimetic hydrazones ONO donor ligands of bioinorganic and medicinal relevance: synthesis via air oxidation of bis(acetylaceto-nato)oxovanadium(IV), characterization, biological activity and 3D molecular modeling," Journal of Molecular Structure, vol. 833, no. 1-3, pp. 133-144, 2007.

[13] A. Butler, M. J. Clague, and G. E. Meister, "Vanadium peroxide complexes," Chemical Reviews, vol. 94, no. 3, pp. 625-638, 1994.

[14] Z. Liu and F. C. Anson, "Schiff base complexes of vanadium(III, IV, V) as catalysts for the electroreduction of $\mathrm{O}_{2}$ to $\mathrm{H}_{2} \mathrm{O}$ in acetonitrile," Inorganic Chemistry, vol. 40, no. 6, pp. 1329-1333, 2001.

[15] A. Salimi, H. Mamkhezri, and S. Mohebbi, "Electroless deposition of vanadium-Schiff base complex onto carbon nanotubes modified glassy carbon electrode: application to the low potential detection of iodate, periodate, bromate and nitrite," Electrochemistry Communications, vol. 8, no. 5, pp. 688-696, 2006.

[16] L. F. Linday and S. E. Livingstone, "Complexes of iron(II), cobalt(II) and nickel(II) with $\alpha$-diimines and related bidentate ligands," Coordination Chemistry Reviews, vol. 2, no. 2, pp. 173-193, 1967.

[17] M. Kuriakose, M. R. P. Kurup, and E. Suresh, "Spectral characterization and crystal structure of 2-benzoylpyridine nicotinoyl hydrazone," Spectrochimica Acta A, vol. 66, no. 2, pp. 353-358, 2007.

[18] S. Das and S. Pal, "Self-assembly of copper(II) complexes with a dibasic tridentate ligand and monodentate N-heterocycles: structural, magnetic and EPR studies," Journal of Molecular Structure, vol. 741, no. 1-3, pp. 183-192, 2005.

[19] M. Carcelli, S. Ianelli, P. Pelagatti et al., "Synthesis and characterization of new lanthanide complexes with hexadentate hydrazonic ligands," Inorganica Chimica Acta, vol. 358, no. 4, pp. 903-911, 2005.

[20] S. Banerjee, A. Ray, S. Sen et al., "Pseudohalide-induced structural variations in hydrazone-based metal complexes: syntheses, electrochemical studies and structural aspects," Inorganica Chimica Acta, vol. 361, no. 9-10, pp. 2692-2700, 2008.

[21] M. Bakir and C. Gyles, "Monosaccharide optical sensor based on ruthenium(II)-bis(bipyridine) of 4-nitrophenyl-di-2pyridyl ketone hydrazone (dpknph), $\left[\mathrm{Ru}\left(\right.\right.$ bipy) ${ }_{2}$ (dpknph) $\mathrm{Cl}_{2}$ ", Journal of Molecular Structure, vol. 753, no. 1-3, pp. 35-39, 2005.

[22] D. Sriram, P. Yogeeswari, and K. Madhu, "Synthesis and in vitro and in vivo antimycobacterial activity of isonicotinoyl hydrazones," Bioorganic and Medicinal Chemistry Letters, vol. 15, no. 20, pp. 4502-4505, 2005.

[23] J. Patole, U. Sandbhor, S. Padhye, D. N. Deobagkar, C. E. Anson, and A. Powell, "Structural chemistry and in vitro antitubercular activity of acetylpyridine benzoyl hydrazone and its copper complex against Mycobacterium smegmatis," Bioorganic and Medicinal Chemistry Letters, vol. 13, no. 1, pp. 51-55, 2003.

[24] J. L. Buss, E. Arduini, and P. Ponka, "Mobilization of intracellular iron by analogs of pyridoxal isonicotinoyl hydrazone $(\mathrm{PIH})$ is determined by the membrane permeability of the ironchelator complexes," Biochemical Pharmacology, vol. 64, no. 12, pp. 1689-1701, 2002.
[25] B. N. Raj and M. R. P. Kurup, "N-2-Hydroxy-4-methoxyacetophenone- $N^{\prime}$-4-nitrobenzoyl hydrazine: synthesis and structural characterization," Spectrochimica Acta A, vol. 66, no. 4-5, pp. 898-903, 2007.

[26] H. H. Monfared, J. Sanchiz, Z. Kalantari, and C. Janiak, "Structure and magnetic properties of a tetranuclear $\mathrm{Cu}_{4} \mathrm{O} 4$ open-cubane in $[\mathrm{Cu}(\mathrm{L})]_{4} \cdot 4 \mathrm{H}_{2} \mathrm{O}$ with $\mathrm{L}^{2-}=(E)-N^{\prime}$-(2-oxy3-methoxybenzylidene)benzohydrazide," Inorganica Chimica Acta, vol. 362, no. 10, pp. 3791-3795, 2009.

[27] H. H. Monfared, Z. Kalantari, M. A. Kamyabi, and C. Janiak, "Synthesis, structural characterization and electrochemical studies of a nicotinamide-bridged dinuclear copper complex derived from a tridentate hydrazone schiff base ligand," Zeitschrift für Anorganische und Allgemeine Chemie, vol. 633, no. 11-12, pp. 1945-1948, 2007.

[28] O. Pouralimardan, A. C. Chamayou, C. Janiak, and H. HosseiniMonfared, "Hydrazone Schiff base-manganese(II) complexes: synthesis, crystal structure and catalytic reactivity," Inorganica Chimica Acta, vol. 360, no. 5, pp. 1599-1608, 2007.

[29] H. H. Monfared, R. Bikas, and P. Mayer, "Homogeneous green catalysts for olefin oxidation by mono oxovanadium $(\mathrm{V})$ complexes of hydrazone Schiff base ligands," Inorganica Chimica Acta, vol. 363, no. 11, pp. 2574-2583, 2010.

[30] R. Bikas, H. H. Monfared, T. Lis, and M. Siczek, "Synthesis, structural characterization and electrochemical studies of an ionic cobalt complex derived from a tridentate hydrazone Schiff base and azide ligands," Inorganic Chemistry Communications, vol. 15, pp. 151-155, 2012.

[31] M. Nielsen and K. V. Gothelf, "Synthesis and catalytic properties of $p$-acylthio(phenylacetylene) ${ }^{n}$ substituted chiral manganese salen complexes," Journal of the Chemical Society, Perkin Transactions, vol. 1, no. 19, pp. 2440-2444, 2001.

[32] A. Altomare, M. C. Burla, M. Camalli et al., "SIR97: a new tool for crystal structure determination and refinement," Journal of Applied Crystallography, vol. 32, no. 1, pp. 115-119, 1999.

[33] P. W. Betteridge, J. R. Carruthers, R. I. Cooper, K. Prout, and D. J. Watkin, "CRYSTALS version 12: software for guided crystal structure analysis," Journal of Applied Crystallography, vol. 36, article 1487, 2003.

[34] C. K. Johnson and M. N. Burnett, ORTEP-III. Report ORNL6895, Oak Ridge National Laboratory, Oak Ridge, Tenn, USA, 1996.

[35] L. J. Farrugia, WINGX, A. MS-Windows System of Programs for Solving, Refining and Analysing Single Crystal X-Ray Diffraction Data for Small Molecules, University of Glasgow, Glasgow, UK, 1999.

[36] L. J. Farrugia, "ORTEP-3 for Windows-a versionof ORTEPIII with a Graphical User Interface (GUI)," Journal of Applied Crystallography, vol. 30, no. 1, p. 565, 1997.

[37] H. H. Monfared, M. Nazari, P. Mayer, M. A. Kamyabi, A. Erxleben, and Z. Asgari, "Synthesis, crystal structure and electrochemical studies of a hydrazone schiff base complex of titanium(IV)," Zeitschrift für Naturforschung B, vol. 64, no. 4, pp. 409-414, 2009.

[38] H. H. Monfared, S. Sadighian, M. A. Kamyabi, and P. Mayer, "Iron(III) aroylhydrazone complexes: structure, electrochemical studies and catalytic activity in oxidation of olefins," Journal of Molecular Catalysis A, vol. 304, no. 1-2, pp. 139-146, 2009.

[39] R. Bikas, H. H. Monfared, C. Kazak, N. B. Arslan, and K. Bijanzad, "(E)-N'-(2-Hydroxybenzylidene)furan-2-carbohydrazide," Acta Crystallographica Section E, vol. 66, no. 8, p. o2015, 2010. 
[40] R. Bikas, P. M. Anarjan, S. W. Ng, and E. R. T. Tiekink, " $N$ '-[(E)]furan-2-carbohydrazide monohydrate," Acta Crystallographica, vol. E68, pp. o413-o414, 2012.

[41] X. S. Tai, J. Yin, M. Y. Hao, and Z. P. Liang, “(E)-N’(5Bromo-2-hydroxy-benzyl-idene)furan-2-carbohydrazide monohydrate," Acta Crystallographica Section E, vol. 63, no. 5, pp. o2144-o2145, 2007.

[42] W. Plass, "Vanadium(V) complexes with flexible and hydrolytically stable amine alcohol ligands and their isomerization in solution: models for the interaction of Vanadium $(V)$ with biogenic ligands," Zeitschrift für Anorganische und Allgemeine Chemie, vol. 623, no. 1-6, pp. 461-477, 1997.

[43] W. Plass, "Oxovanadium(IV) and -(V) complexes with aminoalcohol ligands: synthesis, structure, electrochemical, and magnetic properties," European Journal of Inorganic Chemistry, vol. 1998, pp. 799-805, 1998.

[44] D. L. Pavia, G. M. Lampman, and G. S. Kriz, Introduction to Spectroscopy, Thomson Learning, Albany, NY, USA, 3rd edition, 2001.

[45] G. C. Percy and D. A. Thornton, "N-alkyl salicylaldimine complexes: infrared and PMR spectra of the ligands and vibrational frequencies of their metal(II) chelates," Journal of Inorganic and Nuclear Chemistry, vol. 34, no. 11, pp. 3369-3376, 1972.

[46] G. Kolawole and K. S. Patel, "The stereochemistry of oxovanadium(IV) complexes derived from salicylaldehyde and polymethylenediamines," Journal of the Chemical Society Dalton Transactions, pp. 1241-1245, 1981.

[47] R. T. Conley, Infrared Spectroscopy, Allyn and Bacon, Boston, Mass, USA, 1966.

[48] M. Kuriakose, M. R. Prathapachandra Kurup, and E. Suresh, "Synthesis, spectroscopic studies and crystal structures of two new vanadium complexes of 2-benzoylpyridine containing hydrazone ligands," Polyhedron, vol. 26, no. 12, pp. 2713-2718, 2007.

[49] E. B. Seena, N. Mathew, M. Kuriakose, and M. R. P. Kurup, "Synthesis, spectral and EPR studies of oxovanadium(IV) complexes incorporating tridentate $\mathrm{ONO}$ donor hydrazone ligands: structural study of one oxovanadium(V) complex," Polyhedron, vol. 27, no. 5, pp. 1455-1462, 2008.

[50] A. B. P. Lever, Inorganic Electronic Spectroscopy, Elsevier, Amsterdam, 2nd edition, 1984.

[51] T. Gajda, A. Jancso, S. Mikkola, H. Lonnberg, and H. Sirges, "Crystal structure, solution properties and hydrolytic activity of an alkoxo-bridged dinuclear copper(II) complex, as a ribonuclease model," Journal of the Chemical Society, Dalton Transactions, no. 8, pp. 1757-1763, 2002.

[52] S. Mondal, S. Dutta, and A. Chakravorty, "Synthesis and structure of carboxyl-bonded oxovanadium(V) complexes incorporating $\alpha$-amino acid salicylaldiminates and quinolin-8-olate," Journal of the Chemical Society, Dalton Transactions, no. 7, pp. 1115-1120, 1995.

[53] S. X. Liu and S. Gao, "Synthesis, crystal structure and spectral properties of $\mathrm{VO}$ (acetylacetone benzoylhydrazone)(8quinolinol)," Polyhedron, vol. 17, no. 1, pp. 81-84, 1998.

[54] J. Chakravarty, S. Dutta, A. Dey, and A. Chakravorty, "Synthesis, structure and metal redox of new $\mathrm{VO}^{3+}$ and $\mathrm{VO}^{2+}$ complexes incorporating mixed tridentate-bidentate binding," Journal of the Chemical Society, Dalton Transactions, no. 4, pp. 557-561, 1994.
[55] F. A. Walker, R. L. Carlin, and P. H. Rieger, "ESR studies of coordination to the sixth position of vanadyl acetylacetonate," The Journal of Chemical Physics, vol. 45, no. 11, pp. 4181-4189, 1966. 

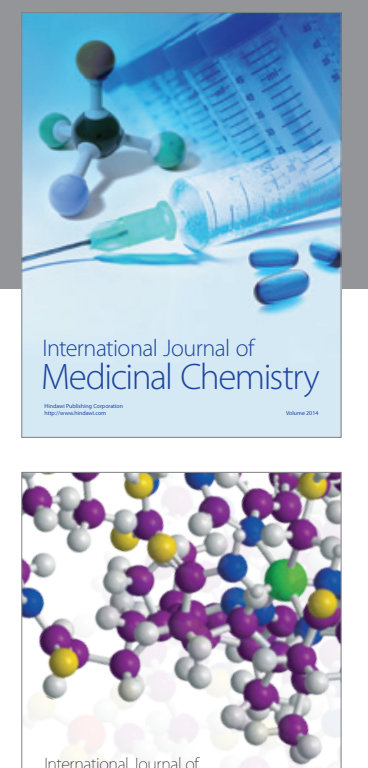

\section{Carbohydrate} Chemistry

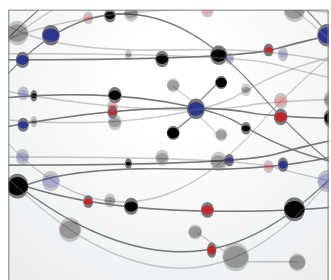

The Scientific World Journal
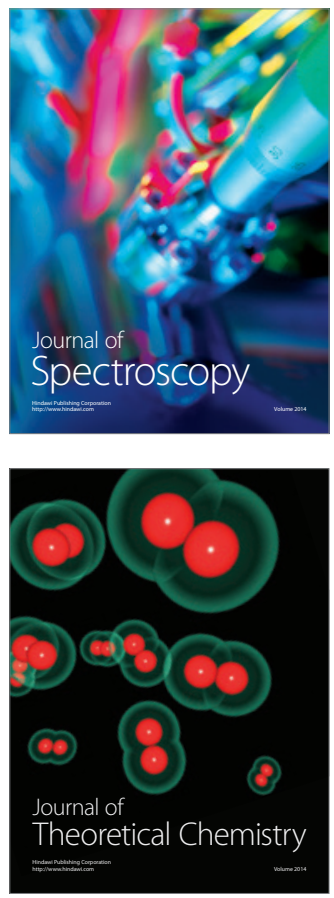
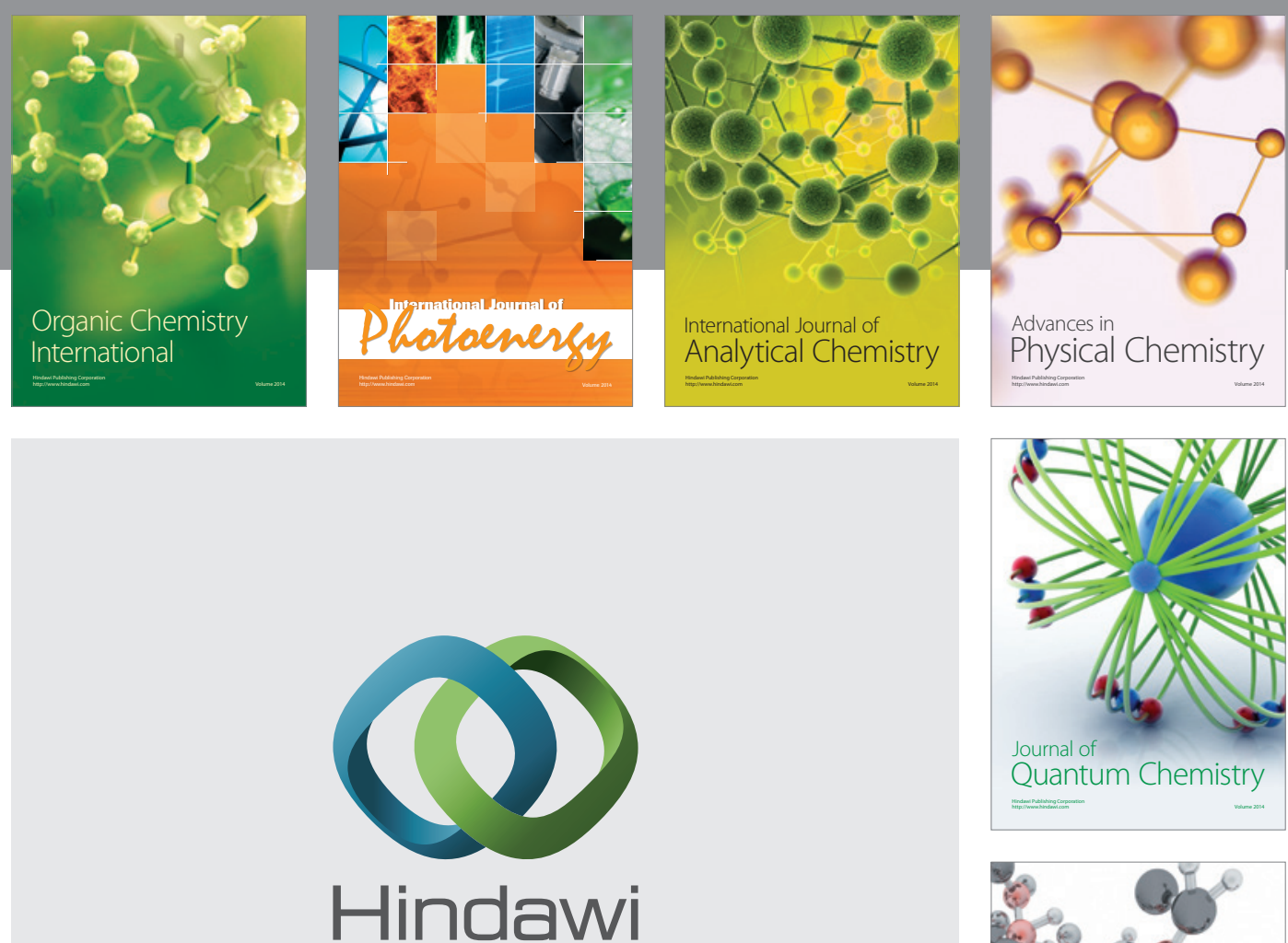

Submit your manuscripts at

http://www.hindawi.com

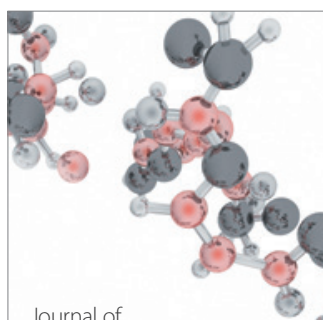

Analytical Methods

in Chemistry

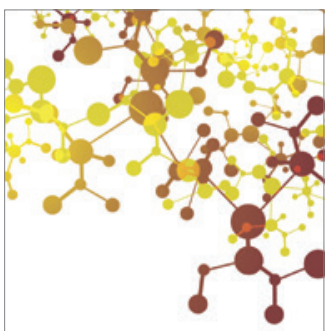

Journal of

Applied Chemistry

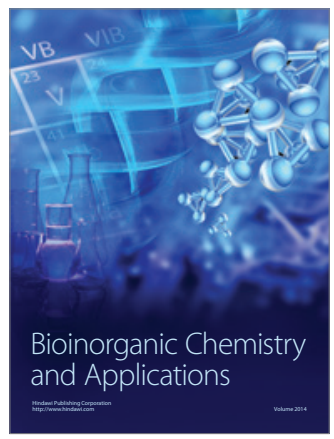

Inorganic Chemistry
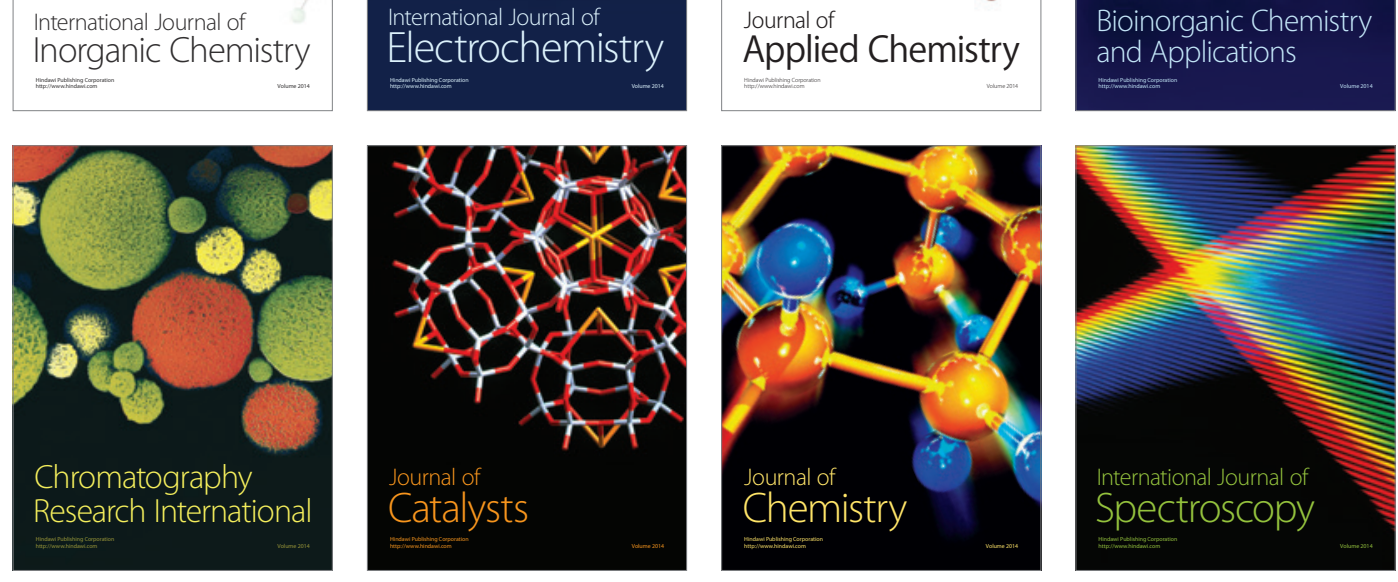